Old Dominion University

ODU Digital Commons

\title{
Dynamic Pair-Breaking Current, Critical Superfluid Velocity, and Nonlinear Electromagnetic Response of Nonequilibrium Superconductors
}

\author{
Ahmad Sheikhzada \\ Old Dominion University, asheikhz@odu.edu \\ Alex Gurevich \\ Old Dominion University, agurevic@odu.edu
}

Follow this and additional works at: https://digitalcommons.odu.edu/physics_fac_pubs

Part of the Elementary Particles and Fields and String Theory Commons

\section{Original Publication Citation}

Sheikhzada, A., \& Gurevich, A. (2020). Dynamic pair-breaking current, critical superfluid velocity, and nonlinear electromagnetic response of nonequilibrium superconductors. Physical Review B, 102(10), (15 pp.), Article 104507 https://doi.org/10.1103/PhysRevB.102.104507

This Article is brought to you for free and open access by the Physics at ODU Digital Commons. It has been accepted for inclusion in Physics Faculty Publications by an authorized administrator of ODU Digital Commons. For more information, please contact digitalcommons@odu.edu. 


\title{
Dynamic pair-breaking current, critical superfluid velocity, and nonlinear electromagnetic response of nonequilibrium superconductors
}

\author{
Ahmad Sheikhzada $\odot^{*}$ and Alex Gurevich ${ }^{\dagger}$ \\ Department of Physics and Center for Accelerator Science, Old Dominion University, Norfolk, Virginia 23529, USA
}

(Received 20 April 2020; revised 19 June 2020; accepted 20 August 2020; published 15 September 2020)

\begin{abstract}
We report numerical calculations of a dynamic pair-breaking current density $J_{d}$ and a critical superfluid velocity $v_{d}$ in a nonequilibrium superconductor carrying a uniform, large-amplitude AC current density $J(t)=$ $J_{a} \sin \Omega t$ with $\Omega$ well below the gap frequency $\Omega \ll \Delta_{0} / \hbar$. The dependencies $J_{d}(\Omega, T)$ and $v_{d}(\Omega, T)$ near the critical temperature $T_{c}$ were calculated from either the full time-dependent nonequilibrium equations for a dirty $s$-wave superconductor or the time-dependent Ginzburg-Landau (TDGL) equations for a gapped superconductor, taking into account the GL relaxation time of the order parameter $\tau_{\mathrm{GL}}$ and the inelastic electron-phonon relaxation time of quasiparticles $\tau_{E}$. We show that both approaches give similar frequency dependencies of $J_{d}(\Omega)$ and $v_{d}(\Omega)$ which gradually increase from their static pair-breaking GL values $J_{c}$ and $v_{c}$ at $\Omega \tau_{E} \ll 1$ to $\sqrt{2} J_{c}$ and $\sqrt{2} v_{c}$ at $\Omega \tau_{E} \gg 1$. Here $J_{d}, v_{d}$ and a dynamic superheating field at which the Meissner state becomes unstable were calculated in two different regimes of a fixed AC current and a fixed AC superfluid velocity induced by the applied AC magnetic field $H=H_{a} \sin \Omega t$ in a thin superconducting filament or a type-II superconductor with a large GL parameter. We also calculated a nonlinear electromagnetic response of a nonequilibrium superconducting state, particularly a dynamic kinetic inductance and a dissipative quasiparticle conductivity, taking into account the oscillatory dynamics of superconducting condensate and the kinetics of quasiparticles driven by a strong AC current. It is shown that an AC current density produces multiple harmonics of the electric field, the amplitudes of the higher-order harmonics diminishing as $\tau_{E}$ increases.
\end{abstract}

DOI: 10.1103/PhysRevB.102.104507

\section{INTRODUCTION}

Mechanisms of the maximum superfluid velocity $v_{c}$ and the DC depairing current density $J_{c}$, which a superconductor can carry in an equilibrium state, have been well established [1]. The first calculations [2] of $v_{c}(T)$ and $J_{c}(T)$ were based on the Ginzburg-Landau (GL) equations near the critical temperature $T \approx T_{c}$. Furthermore, $v_{c}(T)$ and $J_{c}(T)$ have been calculated in the whole temperature range $0<T<T_{c}$ in the BCS model for clean [3-6] and dirty [5,6] superconductors with nonmagnetic and magnetic impurities [7] and taking into account strong electron-phonon coupling in the Eliashberg theory [8]. The DC depairing current densities have been measured for different superconducting materials [9-11]. These issues are closely related to a maximum superheating magnetic field $H_{s}$ which can be sustained by a superconductor in the vortex-free Meissner state. Here $H_{s}(T)$ near $T_{c}$ has been calculated from the GL theory $[12,13]$ and for type-II superconductors with a large GL parameter $\kappa \gg 1$ at $T=0$ [14] and in the entire temperature range $0<T<T_{c}$ both in the clean limit [15] and for arbitrary concentrations of nonmagnetic and magnetic impurities [16]. Nonlinear screening and breakdown of superconductivity in proximity-coupled bilayers under a strong DC magnetic field have been calculated in Refs. [17-20].

\footnotetext{
*asheikhz@odu.edu

${ }^{\dagger}$ gurevich@odu.edu
}

Unlike the static $v_{c}$ and $J_{c}$ in equilibrium, the physics of the dynamic critical superfluid velocity $v_{d}$ and the depairing current density $J_{d}$ at which superconductivity is destroyed in a nonequilibrium state is not well understood. The dynamic $v_{d}$ and $J_{d}$ are controlled by both the nonlinear current pair-breaking effects and a complex kinetics of quasiparticles driven out of equilibrium by a time-dependent electromagnetic field [21]. For an oscillating superflow $J(t)=J_{a} \sin \Omega t$, the dynamic $v_{d}$ and $J_{d}$ depend on the frequency $\Omega$ and the relaxation time constants for the superfluid density $\tau_{\mathrm{GL}}(T)$ and quasiparticles $\tau_{E}(T)$. At $\Omega \ll \Delta / \hbar$ the AC field does not generate new quasiparticles which transfer the absorbed power to phonons. At $k_{B} T \ll \Delta$ this power transfer is mostly limited by an inelastic scattering time of quasiparticles $\tau_{s}(T)$ and a recombination time of Cooper pairs $\tau_{r}(T)$ due to electronphonon collisions [22]:

$$
\tau_{r} \simeq \tau_{1}\left(\frac{T_{c}}{T}\right)^{1 / 2} e^{\Delta / T}, \quad \tau_{s} \simeq \tau_{2}\left(\frac{T_{c}}{T}\right)^{7 / 2},
$$

where $\tau_{1}$ and $\tau_{2}$ are materials constants. Depending on the amplitude $J_{a}$, the distribution function of quasiparticles $f(E, t)$ can either deviate strongly from the Fermi-Dirac distribution $f_{0}(E)$ at $\left(\tau_{r}, \tau_{s}\right) \Omega \gg 1$ or relax to $f_{0}(E)$ at $\left(\tau_{r}, \tau_{s}\right) \Omega \ll 1$. Since both $\tau_{r}(T)$ and $\tau_{s}(T)$ increase as $T$ decreases, nonequilibrium effects become more pronounced at $T \ll T_{c}$. By contrast, $\tau_{\mathrm{GL}}(T)$ increases as $T$ increases and diverges at 
$T=T_{c}[21]$,

$$
\tau_{\mathrm{GL}}(T) \simeq \frac{\pi \hbar}{8 k_{B}\left(T_{c}-T\right)}, \quad T \approx T_{c} .
$$

At $T \ll T_{c}$ the condition $\Omega \tau_{\mathrm{GL}} \lesssim 1$ is satisfied up to $0.1-1$ $\mathrm{THz}$ for most superconductors but breaks down at temperatures very close to $T_{c}$. For instance, at $1 \mathrm{GHz}$ we have $\Omega \tau_{\mathrm{GL}}(T) \simeq 1$ at $T_{c}-T \simeq \pi \hbar \Omega / 8 k_{B} \sim 10^{-2} \mathrm{~K}$.

The dynamics of the condensate at $\Omega \tau_{\mathrm{GL}} \ll 1$ remains nearly quasistatic if the effect of quasiparticles is weak. At $T \ll T_{c}$ the relaxation times $\tau_{s}(T)$ and $\tau_{r}(T)$ increase strongly as the temperature decreases so that $\left(\tau_{r}, \tau_{s}\right) \Omega \gtrsim 1$ while $\Omega \tau_{\mathrm{GL}} \ll 1$, and the AC field can produce highly nonequilibrium quasiparticles. Yet the density of quasiparticles in $s$-wave superconductors at $T \ll T_{c}$ and $\Omega \ll \Delta / \hbar$ is exponentially small as compared to the superfluid density, so the nonequilibrium quasiparticles have only a weak effect on the dynamics of the condensate which reacts almost instantaneously to $J(t)$. In this case, the dynamic $v_{d}$ and $J_{d}$ at $\Omega \ll \Delta / \hbar$ and $T \ll T_{c}$ would be close to the static $v_{c}$ and $J_{c}$ in thermodynamic equilibrium.

The situation changes at $T \approx T_{c}$ where the superfluid density becomes smaller than the density of nonequilibrium quasiparticles which significantly affect the dynamic $v_{d}$ and $J_{d}$ at which superconductivity breaks down. In this work we used both the time-dependent Ginzburg-Landau (TDGL) equations and a full set of nonequilibrium equations for dirty superconductors in a low-frequency $(\Omega \ll \Delta / \hbar)$ field [21,2326] to calculate the dynamic $v_{d}(T, \Omega)$ and $J_{d}(T, \Omega)$ at $T \simeq$ $T_{c}$, where nonequilibrium effects are most pronounced. We consider the case of $\hbar \Omega \ll k_{B} T$ in which the microwave stimulation of superconductivity [27] does not happen, but the AC currents strongly affect the density of states of quasiparticles $[6,28,29]$ and drive them out of equilibrium.

The physics of the dynamic critical velocity is relevant to many applications, for instance, microwave thin film superconducting resonators used in kinetic inductance photon detectors and astrophysical spectroscopy [30,31]. It is also essential for superconducting resonant cavities for particle accelerators, where the breakdown fields close to the thermodynamic superheating field $H_{s}$ have been achieved at very high quality factors $\sim 10^{10}$ at $2 \mathrm{~K}$ in the Meissner state $[32,33]$. These cavities operate at $0.1-3 \mathrm{GHz}$ much lower than the gap frequency $\Delta / h \simeq 0.8 \mathrm{THz}$ for $\mathrm{Nb}$, and the dynamic superheating field $H_{d}$ sets a theoretical limit of the rf breakdown. The dynamic superheating field was measured by Yogi et al. [34] who showed that for $\mathrm{Sn}, \mathrm{Pb}$, and In at $90-300 \mathrm{MHz}$, the breakdown field near $T_{c}$ is close to $H_{s}(T)$. Pulse measurements [35] on $\mathrm{Nb}$ and $\mathrm{Nb}_{3} \mathrm{Sn}$ at $\mathrm{GHz}$ frequencies at $2 \mathrm{~K}<T<T_{c}$ have shown that the field onset of magnetic flux penetration is close to $H_{s}(T)$ for $\mathrm{Nb}$ near $T_{c}$ but is smaller than $H_{S}(T)$ for $\mathrm{Nb}_{3} \mathrm{Sn}$ at lower $T$.

In this work we calculate the dynamic $J_{d}(\Omega, T)$ and a critical phase gradient $Q_{d}(\Omega, T)$ of the order parameter related to $v_{d}$ by $Q_{d}=m v_{d} / \hbar$, where $m$ is the electron mass [1] for a uniform AC superflow at $T \simeq T_{c}$. We focus here on the maximum amplitude of the AC current density $J(t)=J_{a} \sin \Omega t$ which can be sustained in nonequilibrium Meissner states and do not consider nonuniform dissipative states at $J_{a}>J_{d}$ due to proliferation of phase slip centers in narrow filaments [36-38]

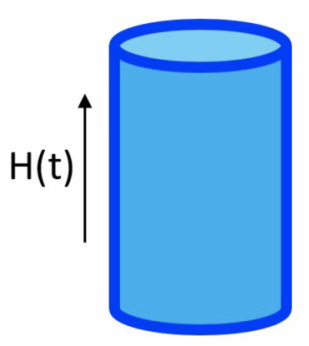

(a) (b)

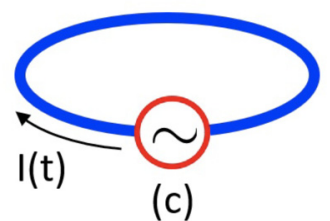

(c)

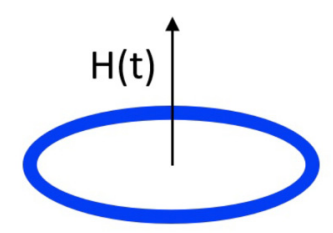

FIG. 1. Geometries for which $Q_{d}(\Omega, T)$ and $J_{d}(\Omega, T)$ are calculated: (a) A thin film cylinder in a parallel AC magnetic field, (b) a thin filament ring in a perpendicular magnetic field, and (c) a thin wire connected to an $\mathrm{AC}$ power supply.

or penetration of vortices in bulk superconductors above the dynamic superheating field. TDGL simulations of thin filaments have shown that $J_{d}$ can approach $\sqrt{2} J_{c}$ at $\Omega \tau_{E} \gg 1$ [38], while numerical simulations of kinetic equations [25,26] have shown [39] that superconductivity can persist during short current pulses with amplitudes above the static $J_{c}$. Yet the calculations of $J_{d}$ and $Q_{d}$, taking into account both the nonlinear current pair-breaking and nonequilibrium kinetics of quasiparticles, have not yet been done. We also calculate a nonlinear electromagnetic response in a nonequilibrium state at $J<J_{d}$ and its manifestations in the nonlinear Meissner effect, kinetic inductance and intermodulation which have been so far investigated in equilibrium superconductors [40-50].

The paper is organized as follows. In Sec. II we specify the main equations and discuss the theoretical assumptions under which the equations have been derived. These equations were solved for a uniform AC superflow in Sec. III, where the dynamic $Q_{d}(T, \Omega)$ and $J_{d}(T, \Omega)$ were calculated. In Sec. IV we address a nonlinear response and calculate the current-dependent kinetic inductance both in equilibrium and nonequilibrium states. The conclusions and broader implications of our results are presented in Sec. V.

\section{MAIN EQUATIONS}

We consider a dirty $s$-wave superconductor exposed to time-dependent electromagnetic potentials $\mathbf{A}(\mathbf{r}, t)$ and $\varphi(\mathbf{r}, t)$. The dynamic $Q_{d}(\Omega, T)$ and $J_{d}(\Omega, T)$ at $T \simeq T_{c}$ are calculated using the equations for the order parameter $\Psi(\mathbf{r}, t)=$ $\Delta \exp (-i \theta)$ and the current density $\mathbf{J}(\mathbf{r}, t)$ along with a kinetic equation for the distribution function of quasiparticles [23-26]. The cases of a fixed AC superfluid velocity $v(t)$ and a fixed AC current density $J(t)$ are investigated. These cases can be realized in the geometries shown in Fig. 1, where a thin film cylinder (a) and a ring filament (b) exposed to the AC magnetic field $H(t)$ correspond to the regime of fixed $v(t)$, whereas a thin wire connected to an AC power supply shown in Fig. 1(c) or a semi-infinite superconductor with $\kappa \gg 1$ corresponds to the regime of fixed $J(t)$. It is assumed that the thickness $d$ of films and filaments is much smaller than the magnetic penetration depth $\lambda_{L}$, so that the induced current 
density is uniform over the cross section. We focus here on the stability of a uniform Meissner state and do not consider thermally activated or quantum proliferation of vortices or phase-slip centers [51-54] and the influence of AC current $[55,56]$ on their dynamics at $J<J_{d}(\Omega, T)$, or the effects of inhomogeneities [57] and current leads on the nucleation of vortices or phase slips. The condition that vortices do not nucleate at $J \sim J_{d}$ requires $d \lesssim \xi(T)$, where $\xi$ is the coherence length. It is also assumed that the magnetic flux threading the samples shown in Fig. 1(a) is much greater than the flux quantum $\phi_{0}$ and the Little-Parks oscillations [1] are washed out. Here the self-field is smaller than the applied field by the factor $d / \lambda_{L} \ll 1$.

The dynamic $Q_{d}(\Omega, T)$ and $J_{d}(\Omega, T)$ for both fixed electric field and fixed current are calculated by first solving the TDGL equations. The TDGL approach is useful to address qualitative mechanisms of destruction of superconductivity by an AC current, even though the TDGL theory, strictly speaking, is not applicable for the calculations of $J_{d}(\Omega, T)$. We then calculate $Q_{d}(\Omega, T)$ and $J_{d}(\Omega, T)$ by solving the full set of dynamic equations of Ref. [24]. Comparing the TDGL results with a more adequate theory of Refs. [23-26] shows the effects of nonequilibrium kinetics of quasiparticles and the extent to which the TDGL approach is applicable. We then proceed with the calculations of the kinetic inductance and the nonlinear electromagnetic response in nonequilibrium states.

\section{A. TDGL equations}

Slow temporal and spatial variations of $\Psi(\mathbf{r}, t)$ and $\mathbf{J}(\mathbf{r}, t)$ in a dirty $s$-wave superconductor at $T \approx T_{c}$ can be described by the TDGL equations $[25,26]$ :

$$
\begin{gathered}
\frac{\pi}{8 T_{c} \epsilon}\left(1+4 \tau_{E}^{2} \Delta^{2}\right)^{-1 / 2}\left(\frac{\partial}{\partial t}+2 i e \varphi+2 \tau_{E}^{2} \frac{\partial \Delta^{2}}{\partial t}\right) \Psi \\
=\left(1-\frac{\Delta^{2}}{\Delta_{0}^{2}}\right) \Psi+\xi^{2}(\nabla-2 i e \mathbf{A})^{2} \Psi, \\
\mathbf{J}=\frac{\pi \sigma_{0}}{4 e T_{c}} \Delta^{2} \mathbf{Q}-\sigma_{0}\left(\nabla \varphi+\frac{\partial \mathbf{A}}{\partial t}\right) .
\end{gathered}
$$

Here $\xi=\left(\pi \hbar D / 8 k_{B} T_{c} \epsilon\right)^{1 / 2}$ is the coherence length, $D=$ $v_{F} l / 3$ is diffusion constant, $v_{F}$ is the Fermi velocity, $l$ is the mean free path, $\epsilon=1-T / T_{c}, \tau_{E}$ is an energy relaxation time due to inelastic scattering of quasiparticles on phonons [21], $\Delta_{0}^{2}=8 \pi^{2} k_{B}^{2} T_{c}^{2} \epsilon / 7 \zeta(3), \sigma_{0}=2 e^{2} D N(0)$ is the normal state conductivity, $N(0)$ is the density of states on the Fermi surface, $-e$ is the electron charge, and $\mathbf{Q}=-\left(\nabla \theta+2 \pi \mathbf{A} / \phi_{0}\right)$ is a gauge-invariant phase gradient. Equations (3) and (4) (in which the units with $\hbar=k_{B}=1$ are used) were derived from the kinetic BCS theory under the condition of local equilibrium, assuming that $\mathbf{Q}(\mathbf{r}, t)$ and $\Delta(\mathbf{r}, t)$ vary slowly over $\xi_{0}$, the diffusion length $L_{E}=\left(D \tau_{E}\right)^{1 / 2}$ and $\tau_{E}[21,25,26]$, where

$$
\tau_{E}=\frac{8 \hbar}{7 \pi \zeta(3) \lambda k_{B} T}\left(\frac{c_{s}}{v_{F}}\right)^{2}\left(\frac{T_{F}}{T}\right)^{2} .
$$

Here $c_{s}$ is the speed of longitudinal sound, $\lambda$ is a dimensionless electron-phonon coupling constant, and $T_{F}=\epsilon_{F} / k_{B}$ is the Fermi temperature. For $\mathrm{Pb}$ we have [58,59] $c_{s} \simeq$ $1.32 \mathrm{~km} / \mathrm{s}, v_{F} \simeq 1830 \mathrm{~km} / \mathrm{s}, T_{F}=1.1 \times 10^{5} \mathrm{~K}, T_{c}=7.3 \mathrm{~K}$, and $\lambda=1.55$, which yields $\tau_{E}^{\mathrm{Pb}}\left(T_{c}\right) \simeq 2.52 \times 10^{-11}$ s. For Al with $c_{s} \simeq 5.1 \mathrm{~km} / \mathrm{s}, v_{F} \simeq 2030 \mathrm{~km} / \mathrm{s}, T_{F}=1.36 \times 10^{5} \mathrm{~K}$, $T_{c}=1.2 \mathrm{~K}$, and $\lambda=0.43$, Eq. (5) gives $\tau_{E}^{\mathrm{Al}}\left(T_{c}\right) \simeq 3.64 \times$ $10^{-7} \mathrm{~s}$.

For a uniform superflow, Eqs. (3) and (4) in the gauge $\varphi=$ 0 can be written in the following dimensionless form:

$$
\begin{gathered}
\left(1+4 \tau^{2} \psi^{2}\right)^{1 / 2} \frac{\partial \psi}{\partial t}=\left(1-q^{2}\right) \psi-\psi^{3}, \\
j=u \psi^{2} q+\frac{\partial q}{\partial t},
\end{gathered}
$$

where $\psi=\Delta / \Delta_{0}, q=Q \xi, \tau=\Delta_{0} \tau_{E} / \hbar, j=J / J_{0}, t$ is in units of $\tau_{\mathrm{GL}}, J_{0}=\sigma_{0} / 2 e \xi \tau_{\mathrm{GL}}$, and $u=\pi^{4} / 14 \zeta(3) \approx 5.79$.

\section{B. Nonequilibrium kinetic equations}

For a uniform current flow, the full set of nonequilibrium kinetic equations [24-26] given in Appendix A can be reduced to a single kinetic equation for the odd in energy $E$ part of the quasiparticle distribution function $f(E, t)$, and dynamic equations for $\psi(t)$ and $j(t)$ :

$$
\begin{gathered}
R_{2} \frac{\partial f}{\partial E} \frac{\partial \psi}{\partial t}+N_{1}\left(\frac{\partial}{\partial t}+\frac{s}{2 \tau}\right) \delta f=\frac{N_{2} R_{2}}{s} \frac{\partial f}{\partial E} \frac{\partial q^{2}}{\partial t}, \\
\frac{\partial \psi}{\partial t}-\frac{1}{\epsilon} \int_{0}^{\infty} R_{2} \delta f d E=\left(1-q^{2}\right) \psi-\psi^{3} \\
j=u \psi^{2} q+\frac{\partial q}{\partial t} \int_{0}^{\infty}\left(N_{1}^{2}+N_{2}^{2}\right) \frac{\partial f}{\partial E} d E \\
+2 q s \int_{0}^{\infty} N_{2} R_{2} \delta f d E, \quad s=(u / \epsilon)^{1 / 2} .
\end{gathered}
$$

Here $\delta f(E, t)=f(E, t)-f_{0}(E), \quad f_{0}=\tanh (E / 2 T)$, the quasiparticle energy $E$ and temperature $T$ are in units of $\Delta_{0}$, and the scaling factor $(u / \epsilon)^{1 / 2}=2 \tau_{\mathrm{GL}} \Delta_{0} / \hbar$ results from the same normalization of the parameters as in Eqs. (6) and (7). If $\Omega \tau_{\mathrm{GL}} \ll 1$ the spectral functions $N_{1}, N_{2}, R_{1}$, and $R_{2}$ are defined by the normal $\alpha(E)=N_{1}(E)+i R_{1}(E)$ and anomalous $\beta(E)=N_{2}(E)+i R_{2}(E)$ Green's functions which satisfy the quasistatic Usadel equation for 1D current flow $[25,26]$ :

$$
\left(\frac{1}{2 \tau}-i E\right) \beta+\frac{q^{2}}{2} \alpha \beta=\psi \alpha,
$$

where $\alpha^{2}+\beta^{2}=1$. Equation (11) reduces to a quartic equation for $\alpha$, the solutions of which are given in Appendix A. The term $1 / 2 \tau$ in Eq. (11) defines a finite quasiparticle lifetime due to scattering on phonons, resulting in subgap states at $|E|<\psi$. We do not consider here other contributions to the subgap states [60-62].

We solved the integro-differential Eqs. (8)-(10) numerically using the method of lines [63]. By discretizing the energy, Eqs. (8)-(10) were reduced to coupled ordinary differential equations in time which were solved by the Adams-Bashforth-Moulton method [64] with the error tolerances below $10^{-6}$. Results of the calculations of the dimensionless $j_{d}=J_{d} / J_{0}$ and $q_{d}=Q_{d} \xi$ as functions of the dimensionless frequency $\omega=\Omega \tau_{\mathrm{GL}}$ and the quasiparticle relaxation time $\tau=\tau_{E} \Delta_{0} / \hbar$ are given below. 


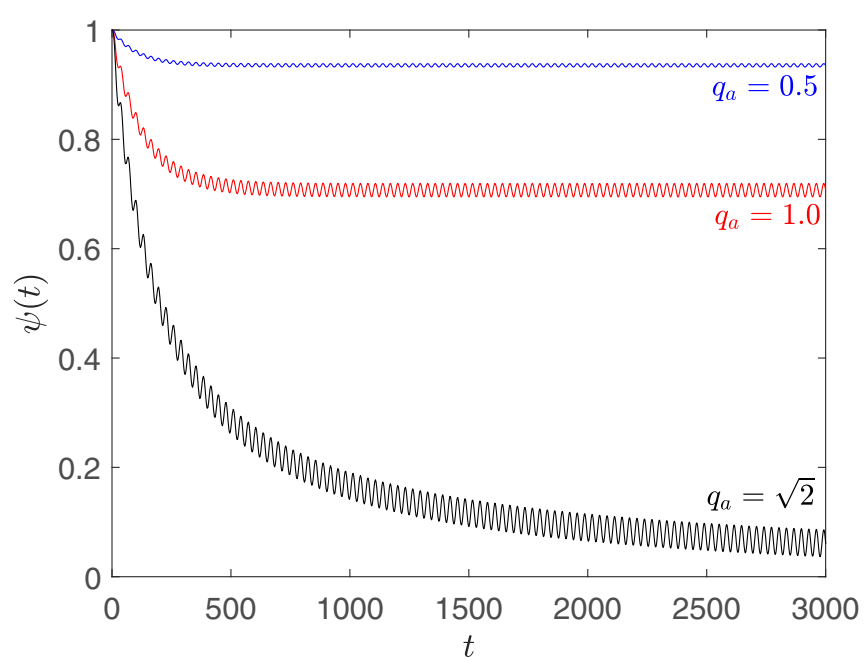

FIG. 2. Dynamics of $\psi(t)$ calculated at $q=q_{a} \sin \omega t, \tau=100$, and $\omega=0.1$. Here $\psi(t)$ eventually vanishes at $q_{a}=\sqrt{2}$.

\section{DYNAMIC PAIR-BREAKING CURRENT}

\section{A. TDGL results}

The stationary Eqs. (6) and (7) have the solution $\psi=0$ at $q>1$ and $\psi=\sqrt{1-q^{2}}$ at $q<1$. Stability of this solution with respect to small perturbations $\delta \psi(t)$ and $\delta q(t)$ depends on the way by which the superflow is generated. In the regime of fixed $q$ the stationary solution $\psi(q)$ is stable in the whole region of $q<q_{c}=1$, but in the regime of fixed $j$ the solution $\psi(q)$ is stable if $q$ is smaller than $q_{c}=1 / \sqrt{3}$ at which $j=u q\left(1-q^{2}\right)$ reaches maximum [1,2]. This gives the GL depairing current density $j_{c}=2 u / 3 \sqrt{3}$ above which $\psi(j)$ drops from $\psi\left(j_{c}\right)=\sqrt{2 / 3}$ to zero.

\section{Fixed $Q(t)$}

Figure 2 shows $\psi(t)$ calculated from Eq. (6) with $q(t)=$ $q_{a} \sin \omega t$ at $\omega=\Omega \tau_{\mathrm{GL}}=0.1, \tau=100$, and the initial condition $\psi(0)=1$. Here $\psi(t)$ relaxes after a transient period $t \gtrsim$ $\sqrt{1+4 \tau^{2}}$ to an oscillating steady state with a nonzero mean $\langle\psi\rangle$ if $q_{a}<q_{d}(\omega, T)$ or to the normal state with $\psi(t)=0$ at $t \gg 1$ if $q_{a}>q_{d}(\omega, T)$. The mean $\left\langle\psi\left(q_{a}\right)\right\rangle$ decreases with $q_{a}$ and vanishes at $q_{a}=q_{d}$.

The calculated dependencies of $q_{d}$ on $\omega$ and $\tau$ are shown in Fig. 3. Here $q_{d}(\tau)$ at $\omega=0.01$ increases from $q_{d}(0) \approx$ 1.097 at $\tau=0$ to $q_{d}(\tau) \rightarrow \sqrt{2}$ at $\tau \gg 1$. At higher frequency $\omega=0.1$, the dynamic $q_{d}(\tau)$ is nearly equal to $\sqrt{2}$ at all $\tau$. However, if $\tau$ is fixed but the frequency changes, $q_{d}(\omega)$ varies from $q_{c}=1$ at $\omega=0$ to $q_{d}(\omega) \rightarrow \sqrt{2}$ at $\omega \sqrt{1+4 \tau^{2}} \gg 1$. The universal value of $q_{d}=\sqrt{2}$ is achieved at $\omega \tau \gtrsim 1$, that is, for $\Omega$ exceeding a crossover frequency $\Omega_{c} \simeq \hbar / \tau_{\mathrm{GL}} \Delta_{0} \tau_{E}$ given by

$$
\Omega_{c} \simeq \frac{k_{B}}{\Delta_{0} \tau_{E}}\left(T_{c}-T\right) \sim \frac{k_{B} T^{3}}{\hbar T_{D}^{2}} \sqrt{1-\frac{T}{T_{c}}}
$$

where $T_{D}$ is the Debye temperature. Here $\Omega_{c}(T)$ vanishes at $T_{c}$, reaches maximum $\Omega_{m}=\Omega_{c}\left(6 T_{c} / 7\right)$ at $T / T_{c} \approx 0.86$ and decreases with $T$ at $T<0.8 T_{c}$, as shown in Fig. 4 .
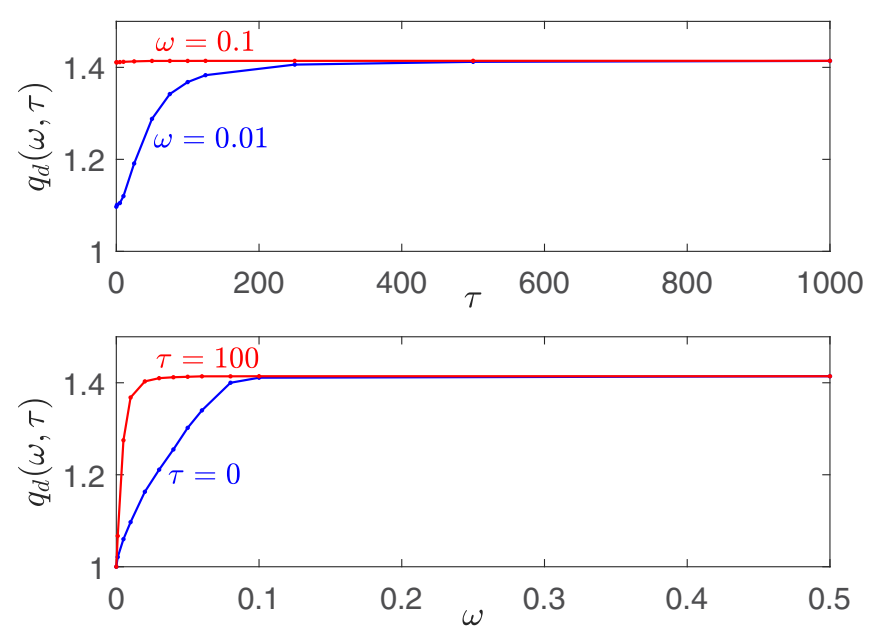

FIG. 3. The calculated dependencies of $q_{d}$ on $\tau$ (top) and $\omega$ (bottom). Here $q_{d} \rightarrow \sqrt{2}$ at $\omega \tau \gtrsim 1$.

The increase of $Q_{d}(\Omega, T)$ at $\Omega \gtrsim \Omega_{c}(T)$ by the factor $\sqrt{2}$ can be understood as follows. As follows from Fig. 2, $\psi(t)$ oscillates rapidly around a mean $\langle\psi\rangle$. Here $\langle\psi\rangle \simeq \sqrt{1-\left\langle q^{2}\right\rangle}$ is determined by Eq. (6) with the time-averaged $\left\langle q^{2}(t)\right\rangle=q_{a}^{2} / 2$ so $\langle\psi\rangle$ vanishes at $q_{a}=\sqrt{2}$. A small-amplitude AC correction $\delta \psi(t)$ was calculated in Appendix B. The superconducting state remains stable in the whole region $0<q_{a}<q_{d}$.

The temperature dependence of $Q_{d}(\Omega, T)$ shown in Fig. 5 is affected by the ratio $\Omega / \Omega_{c}(T)$. If $\Omega>\Omega_{m}=\Omega_{c}\left(6 T_{c} / 7\right)$ (see Fig. 4), the dynamic $Q_{d}(T) \rightarrow \sqrt{2} / \xi(T)$ has the same temperature dependence as the static $Q_{c}=1 / \xi(T)$. However, if $\Omega \ll \Omega_{m}$, we obtain that $Q_{d}(T) \rightarrow \xi_{0}^{-1} \sqrt{2\left(1-T / T_{c}\right)}$ at $T$ close to $T_{c}$ and crosses over to the static $Q_{c}(T)$ at lower $T$. There is also a range of frequencies $\Omega<\Omega_{m}$ but $\Omega \gtrsim \Omega_{c}\left(T_{c} / 2\right)$ (see Fig. 4) in which $Q_{d}(T)$ evolves from $\sqrt{2} Q_{c}(T)$ at $T \rightarrow T_{c}$ to $Q_{d} \simeq Q_{c}(T)$ at $T \lesssim 0.8 T_{c}$ and back to $\simeq \sqrt{2} Q_{c}(T)$.

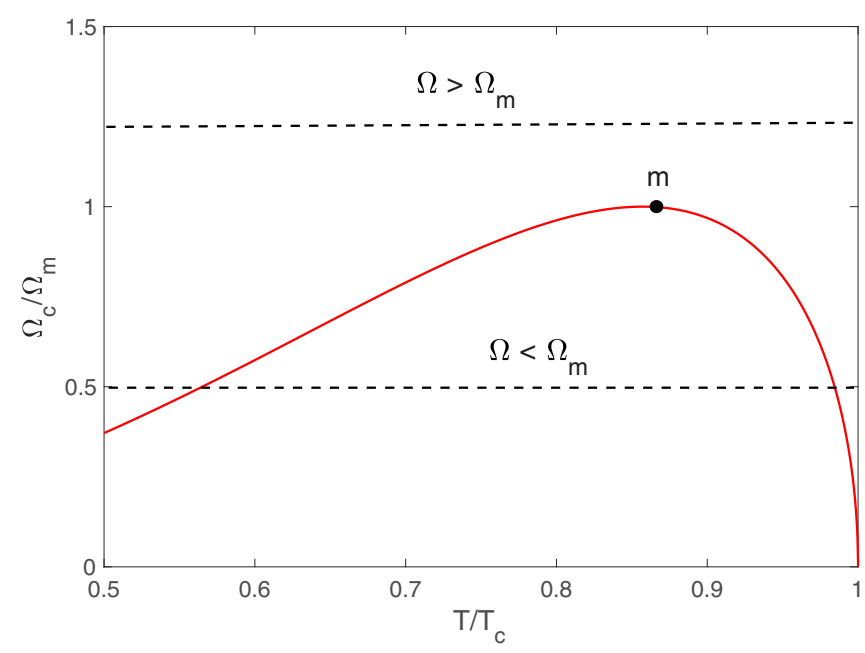

FIG. 4. Temperature dependence of $\Omega_{c}(T)$. The dashed lines show the levels of fixed $\Omega$ at $\Omega>\Omega_{m}$ and $\Omega<\Omega_{m}$, where $\Omega_{m}$ is the maximum value of $\Omega_{c}(T)$ corresponding to the point $m$. 


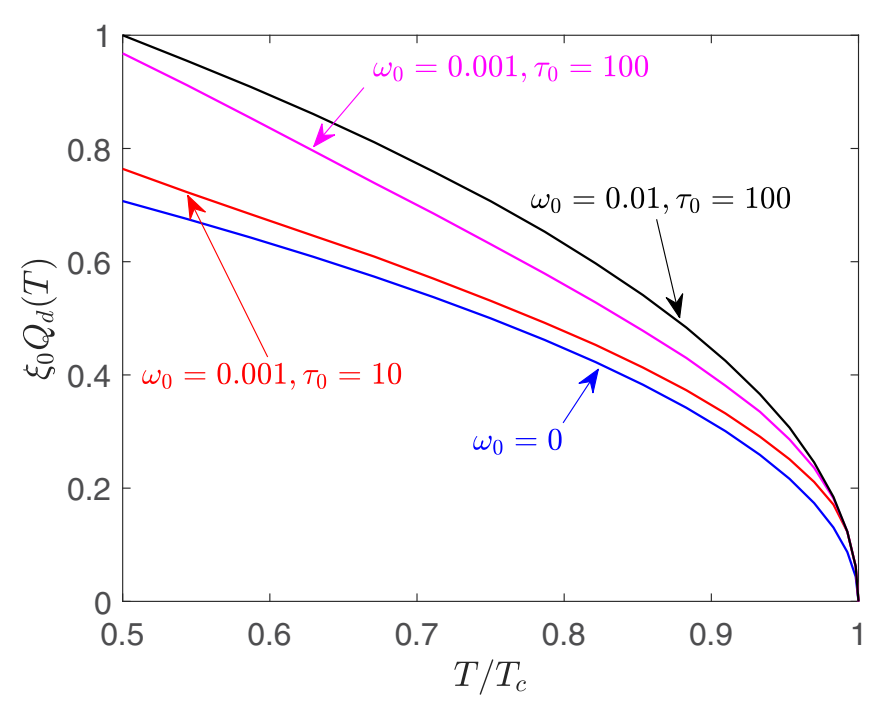

FIG. 5. $Q_{d}(T)$ calculated for different values of $\omega_{0}=$ $\pi \hbar \Omega / 8 k_{B} T_{c}$ and $\tau_{0}=\tau_{E}\left(T_{c}\right) \Delta_{0}(0)$, where $\Delta_{0}^{2}(0)=8 \pi^{2} T_{c}^{2} / 7 \zeta(3)$. Here the dynamic $Q_{d}=\sqrt{2\left(1-T / T_{c}\right)} / \xi_{0}$ at $\Omega \gg \Omega_{c}(T)$ has the same temperature dependence as the static $Q_{c}=\sqrt{1-T / T_{c}} / \xi_{0}$. If $\Omega \sim \Omega_{c}(T)$ the behavior of $Q_{d}(T)$ is affected by the temperature dependence of $\tau_{E}(T)$, as shown for the case of $\omega_{0}=0.001$ and $\tau_{0}=100$.

\section{Fixed $J(t)$}

We calculated $\psi(t)$ at a fixed $j(t)=j_{a} \sin \omega t$ by solving the coupled Eqs. (6) and (7). The GL DC depairing current density $j_{c}=2 u / 3 \sqrt{3} \approx 2.228$ is reached at $q=1 / \sqrt{3}$ and $\psi^{2}=2 / 3$, while at $q>1 / \sqrt{3}$ the superconducting state becomes unstable and $\psi(q)$ vanishes abruptly [1]. This feature is characteristic of the AC current as well, which makes it different from the regime of fixed $q(t)$. For instance, Fig. 6 shows $\psi(t)$ calculated at $\tau=10$ and $\omega=0.1$. At $j_{a}=1.38 j_{c}$

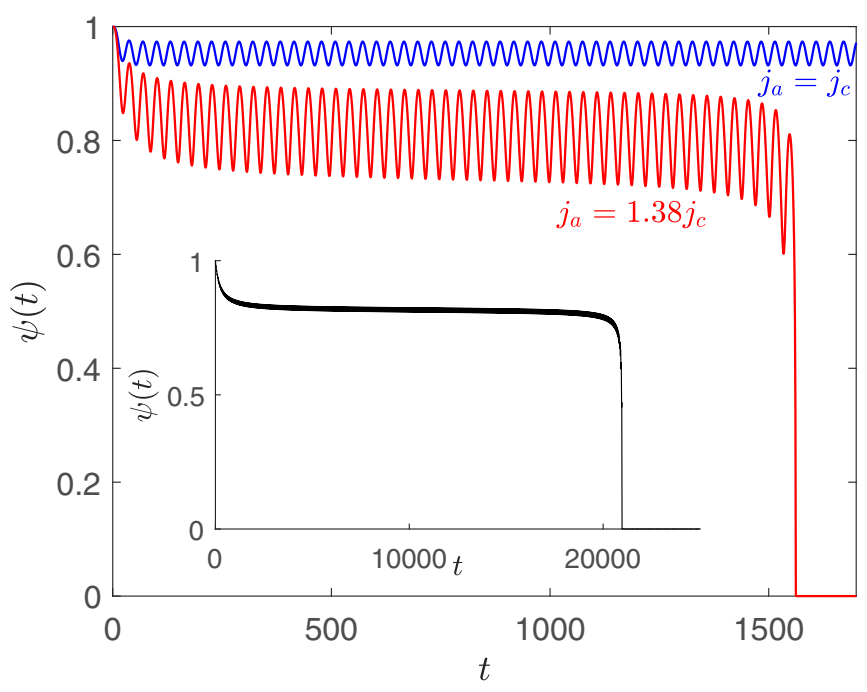

FIG. 6. Dynamics of $\psi(t)$ calculated at $j=j_{a} \sin \omega t, \omega=0.1$, $\tau=10$, and different amplitudes $j_{a}$. At $j_{a}=j_{c}$, the superconducting state still exists, but once $j_{a}$ reaches the dynamic pair-breaking current $j_{d}=1.38 j_{c}, \psi(t)$ vanishes. The inset shows $\psi(t)$ calculated at $\tau=100$ at $j_{a}=\sqrt{2} j_{c}$ and $\omega=0.1$.
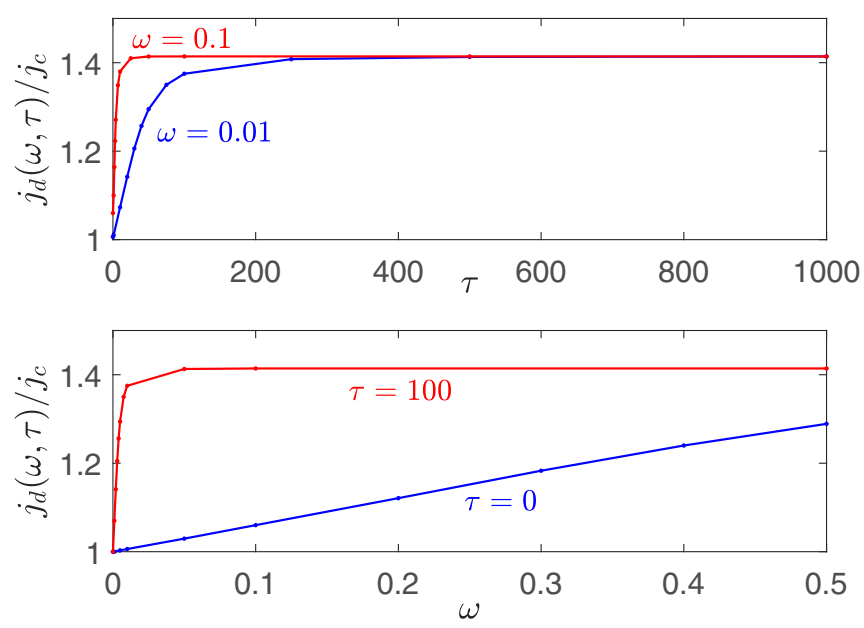

FIG. 7. Dynamic pair-breaking current $j_{d}$ as a function of $\tau$ (top) and $\omega$ (bottom). Here $j_{d}(\omega, \tau) \rightarrow \sqrt{2} j_{c}$ at $\omega \tau \gg 1$.

the order parameter abruptly vanishes after a transient period. For large $\tau$, this transition to the normal state occurs at $j_{a}=$ $\sqrt{2} j_{c}$, as shown in the inset for $\tau=100$ and $\omega=0.1$. Here the dynamic pair-breaking current $j_{d}(\omega, \tau)$ shown in Fig. 7 exhibits similar dependencies on $\omega$ and $\tau$ as $q_{d}(\omega, \tau)$ at a fixed $q(t)$. If $\omega \tau \gtrsim 1$ both the dynamic $j_{d}(\omega, \tau)$ and $q_{d}(\omega, \tau)$ are larger by the factor $\sqrt{2}$ than their respective GL values.

The temperature dependence of $J_{d}(\Omega, T)$ is affected by the temperature dependencies of $\tau(T)$ and $\Omega_{c}(T)$. At $T \rightarrow T_{c}$ and $\Omega \gtrsim \Omega_{c}(T)$ the dynamic pair-breaking current $J_{d}$ is $\sqrt{2}$ times larger than the static $J_{c}(T)$ and is independent of $\tau$. As $T$ decreases $J_{d}(\Omega, T)$ can evolve to $J_{c}(T)$ at temperatures for which $\Omega \lesssim \Omega_{c}(T)$. This behavior of $J_{d}(\Omega, T)$ is illustrated in Fig. 8.

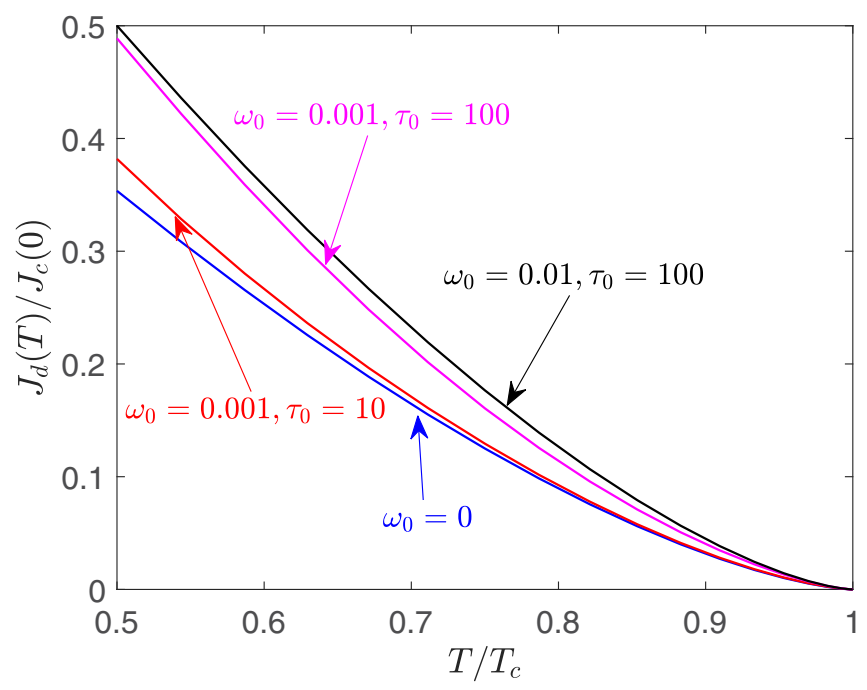

FIG. 8. $J_{d}(T)$ calculated for different values of $\omega_{0}=\pi \hbar \Omega / 8 k_{B} T_{c}$ and $\tau_{0}=\tau_{E}\left(T_{c}\right) \Delta_{0}(0)$, where $\Delta_{0}^{2}(0)=8 \pi^{2} T_{c}^{2} / 7 \zeta(3)$. Here the dynamic $J_{d}=\sqrt{2} J_{c}(0)\left(1-T / T_{c}\right)^{3 / 2}$ at $\Omega \gg \Omega_{c}(T)$ has the same temperature dependence as the static $J_{c}=J_{c}(0)\left(1-T / T_{c}\right)^{3 / 2}$. At $\Omega \sim \Omega_{c}(T)$ the behavior of $J_{d}(T)$ is affected by the temperature dependence of $\tau_{E}(T)$, as shown for the case of $\omega_{0}=0.001$ and $\tau_{0}=100$. 


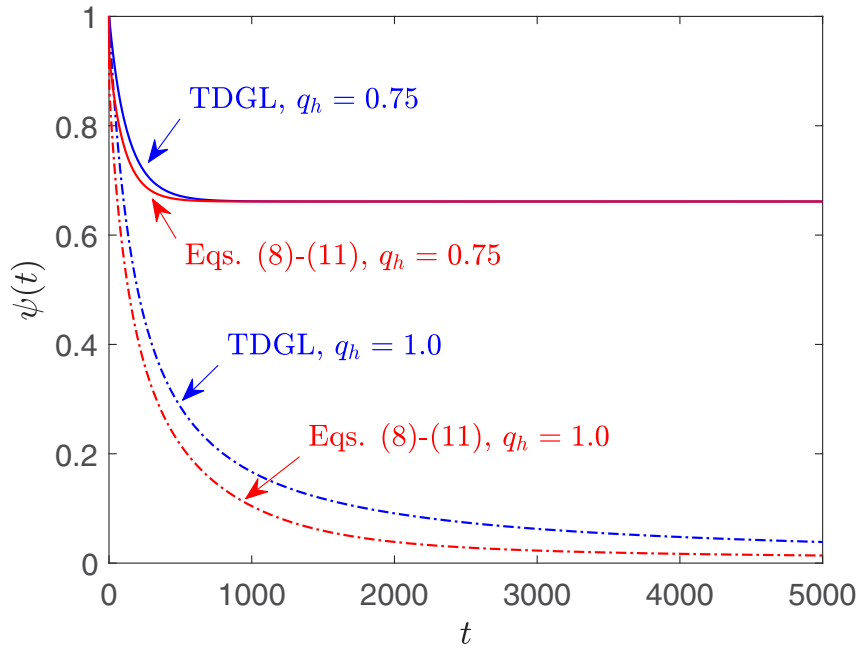

FIG. 9. Comparison of $\psi(t)$ calculated from the TDGL equation (6) and the full nonequilibrium equations (8)-(11) for $q(t)=$ $q_{h} \tanh t$ at $q_{h}=0.75$ and $q_{h}=1$. Here we took $\tau(T)=100$ and $T=0.9 T_{c}$.

\section{B. $Q_{d}(T, \Omega)$ and $J_{d}(T, \Omega)$ calculated from the full set of nonequilibrium equations}

The TDGL calculations of $q_{d}(T, \omega)$ and $j_{d}(T, \omega)$ give a qualitative picture of dynamic pair breaking, although Eqs. (6) and (7) are not really applicable at $J \simeq J_{d}$. Indeed, the dynamic terms in Eqs. (6) and (7) were derived from the BCS kinetic theory, assuming weak pair breaking and local equilibrium in which $Q \xi \ll 1$ and $\Delta(\mathbf{r}, t)$ varies slowly over the diffusion length $L_{E}=\left(D \tau_{E}\right)^{1 / 2}$ and the energy relaxation time $\tau_{E}[25,26]$. Those conditions break down at $Q \simeq Q_{c} \sim \xi^{-1}$ and $\Omega \gtrsim \tau_{\mathrm{GL}}^{-1}$, so in this section we calculate $\psi(t), q_{d}(T, \omega)$, and $j_{d}(T, \omega)$ from Eqs. (8)-(10) which take into account both the dynamic current pair breaking and nonequilibrium kinetics of quasiparticles.

Consider first solutions of Eqs. (8)-(11) at $\tau(T)=100$ and $T=0.9 T_{c}$ for a superflow $q(t)=q_{h} \tanh t$ which was gradually turned on at $t=0$. As shown in Fig. 9, the qualitative behavior of $\psi(t)$ calculated from Eqs. (8) and (9) turns out to be similar to that of TDGL, except that the nonequilibrium integral term in Eq. (9) accelerates relaxation of $\psi(t)$ at $q_{h} \simeq 1$. In both cases superconductivity is destroyed at $q_{h}=1$.

Shown in Fig. 10 are snapshots of a nonequilibrium part of the distribution function $\delta f(E, t)$ induced by the stepwise $q(t)$. Here the magnitude of $\delta f(E, t)$ calculated at $\tau=100$ increases as $q_{h}$ increases but remains relatively small up to $q_{h}=1$. As the quasiparticle relaxation time $\tau$ increases, the magnitude of $\delta f(E, t)$ also increases. The peak in $\delta f(E, t)$ shifts to lower energies as $q_{h}$ increases, consistent with the decrease of the quasiparticle gap due to the DC current pair breaking.

\section{Fixed $Q(t)$}

Solutions of Eqs. (8) and (9) with $q(t)=q_{a} \sin \omega t$ are shown in Fig. 11 along with the TDGL results obtained for the same input parameters. At $q_{a}=1$ the order parameters $\psi(t)$ oscillate around nearly the same mean values $\langle\psi\rangle$ but

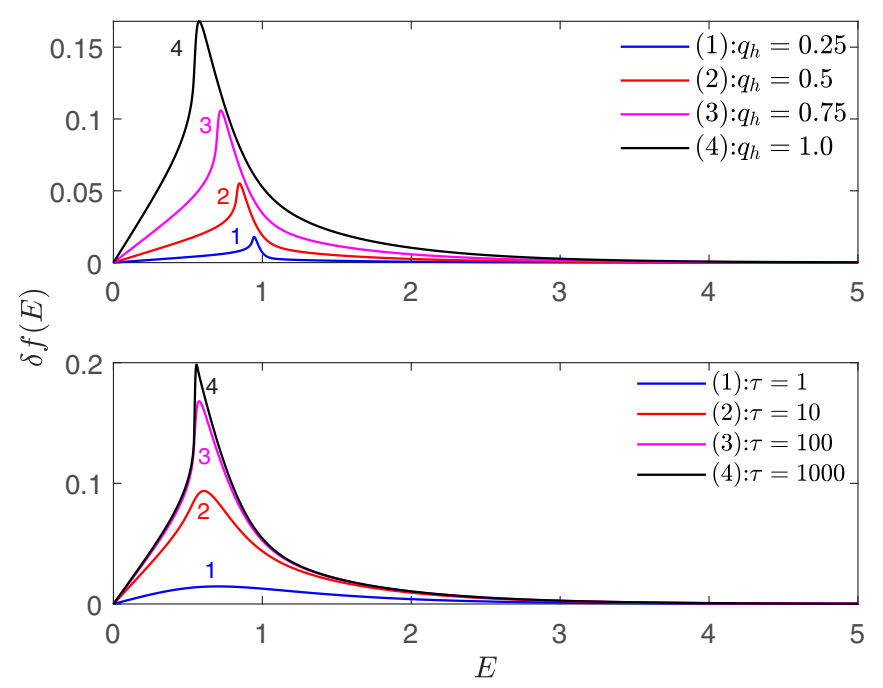

FIG. 10. The nonequilibrium correction $\delta f(E)$ at the times when the magnitude $\delta f(E, t)$ reaches maximum after the stepwise increase of $q(t)$. Taking $T=0.9 T_{c}$, here the top panel shows $\delta f(E, t)$ calculated for different $q_{h}$ at $\tau=100$ and the bottom panel shows $\delta f(E, t)$ calculated for different values of $\tau$ at $q_{h}=1$.

the amplitude of oscillations $\delta \psi(t)$ calculated from Eqs. (8) and (9) is noticeably larger than the TDGL $\delta \psi(t)$. Relaxation of $\psi(t)$ from the initial value $\psi(0)=1$ to the steady-state oscillations described by Eqs. (8) and (9) is also faster than the TDGL transient time, consistent with the above results for $q(t)=q_{h} \tanh t$ shown in Fig. 9. These features become more pronounced at the dynamic critical momentum $q_{d} \simeq \sqrt{2}$ at $\omega \tau \gg 1$, where the amplitudes of oscillations $\delta \psi(t)$ grow significantly larger so that $\psi(t)$ touches zero but then recovers. Yet, despite a rather different dynamics of $\psi(t)$ described by Eqs. (8) and (9) and the TDGL equations, superconductivity gets destroyed at the same critical value $q_{d} \rightarrow \sqrt{2}$ at $\tau=100$ and $\omega=0.1$ in both cases. The calculated dependencies of

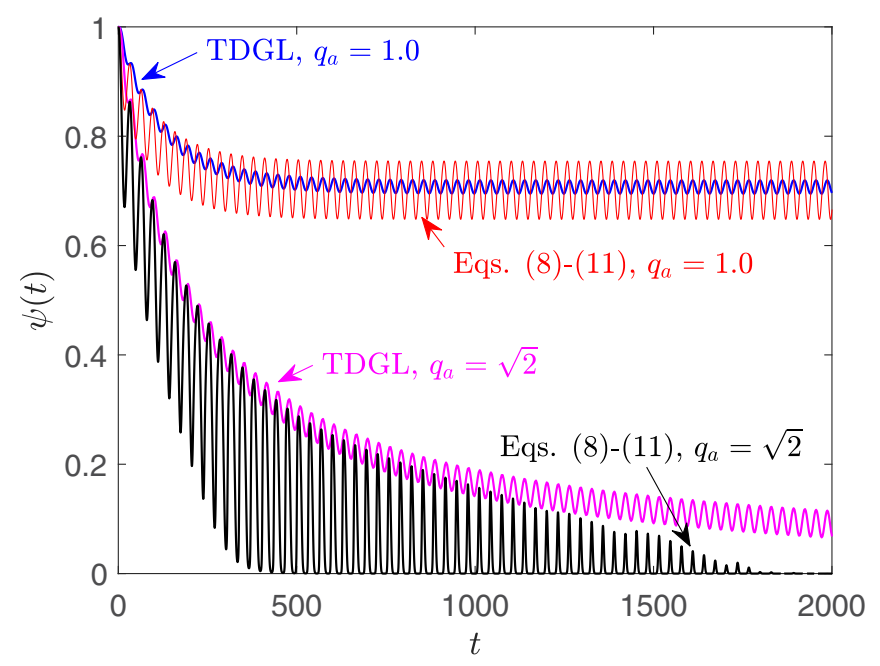

FIG. 11. Comparison of $\psi(t)$ calculated from the TDGL equations and Eqs. (8) and (9) for $q(t)=q_{a} \sin \omega t, \tau=100, \omega=0.1$, and $T=0.9 T_{c}$. 

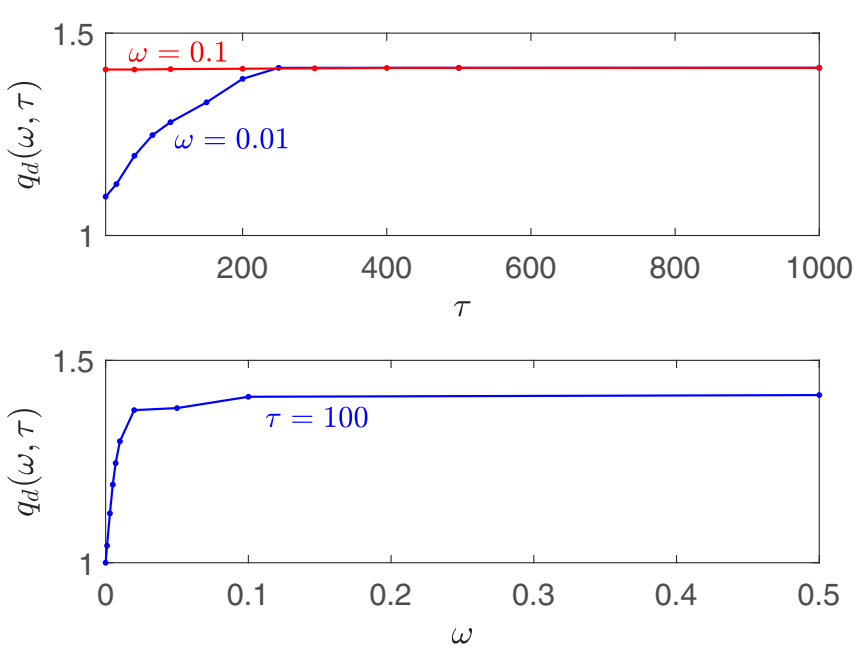

FIG. 12. Dynamic $q_{d}(\omega, \tau)$ as functions of $\tau$ (top) and $\omega$ (bottom) calculated from Eqs. (8) and (9) at $T=0.9 T_{c}$.

$q_{d}$ on $\tau$ and $\omega$ shown in Fig. 12 appear similar to the TDGL results shown by Fig. 3 .

Our solutions of Eqs. (8) and (9) have revealed a dynamic state in which $\psi(t)$ periodically vanishes but then recovers to $\psi(t) \sim 1$. This state appears as the frequency decreases, as shown in Fig. 13. For instance, in the case of $\omega=0.1$ and $\tau=10$ shown in the top panel Fig. 13, $\psi(t)$ drops down to $\sim 2 \times 10^{-3}$ at the minimum but remains finite. As $\psi(t)$ goes through the minimum the amplitude of $\delta f(E, t)$ decreases and changes sign. However, at $\omega=0.01$ in the bottom panel, $\psi(t)$ at the minimum drops below the numerical tolerance level of $\sim 10^{-7}$ during a significant portion of the AC period. This case corresponds to a true transition to the normal state with $\psi=0$ in which all terms in Eq. (9) vanish and Eq. (8) describes an exponential relaxation of $\delta f(E, t) \propto \exp (-t s / 2 \tau)$ until the superconductivity recovers as $q(t)$ decreases. This behavior is physically transparent: at very low frequencies the quasistatic
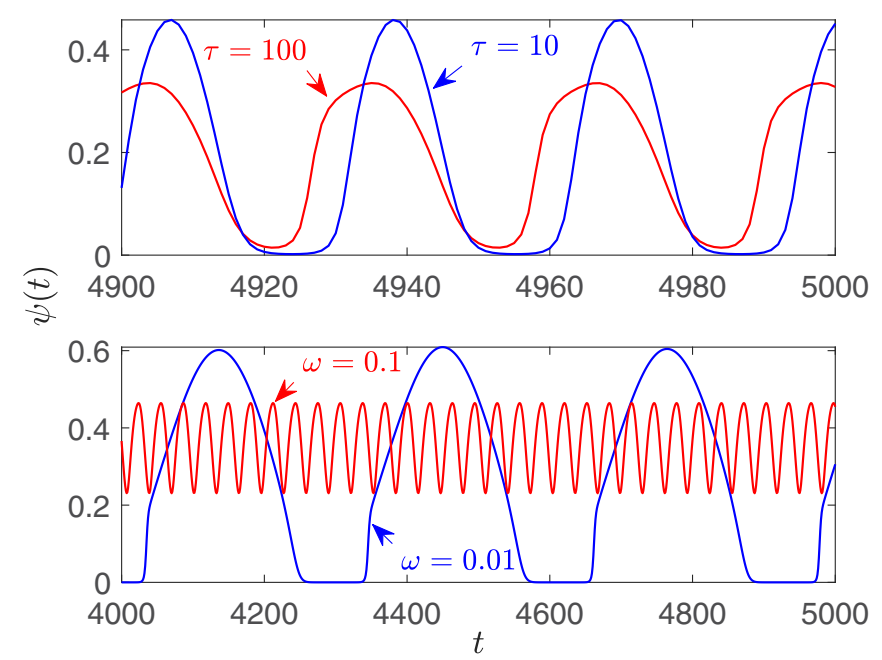

FIG. 13. Steady state oscillations of $\psi(t)$ calculated from Eqs. (8) and (9) at $T=0.9 T_{c}$ with $q=q_{a} \sin \omega t$ for different $\tau$ at $\omega=0.1$ and $q_{a}=1.35$ (top) and different $\omega$ at $\tau=100$ and $q_{a}=$ 1.30 (bottom).

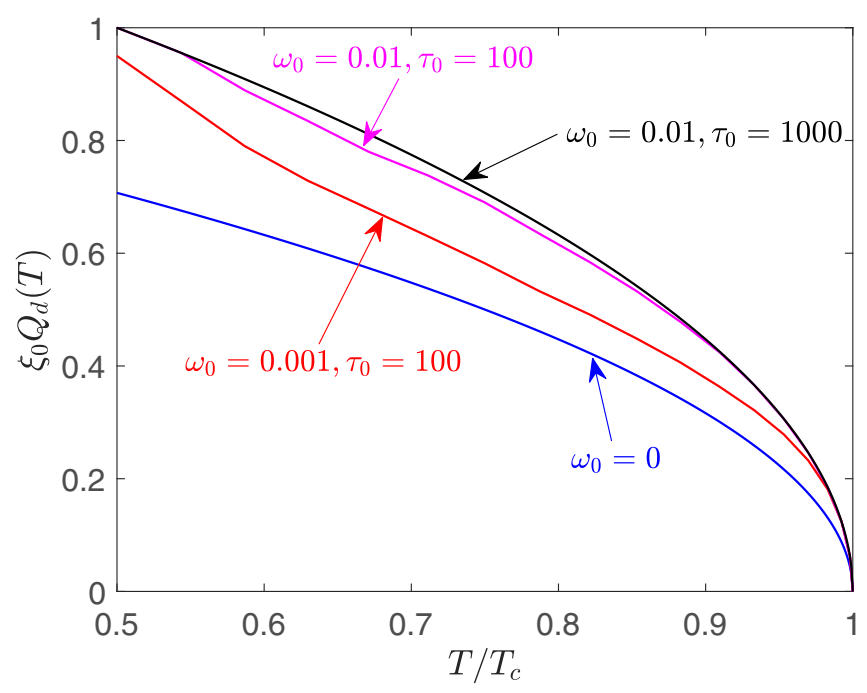

FIG. 14. $Q_{d}(T)$ calculated from Eqs. (8) and (9) for different values of $\omega_{0}=\pi \hbar \Omega / 8 k_{B} T_{c}$ and $\tau_{0}=\tau_{E}\left(T_{c}\right) \Delta_{0}(0)$, where $\Delta_{0}^{2}(0)=8 \pi^{2} T_{c}^{2} / 7 \zeta(3)$. The dynamic $Q_{d}=\sqrt{2\left(1-T / T_{c}\right)} / \xi_{0}$ at $\Omega \gg \Omega_{c}(T)$ has the same temperature dependence as the static $Q_{c}=$ $\sqrt{1-T / T_{c}} / \xi_{0}$. If $\Omega \sim \Omega_{c}(T)$ the behavior of $Q_{d}(T)$ is affected by the temperature dependence of $\tau_{E}(T)$, as shown for the case of $\omega_{0}=0.001$ and $\tau_{0}=100$.

$\psi(t)$ is determined by the instantaneous $q(t)=q_{a} \sin \omega t$, resulting in periodic transitions to the normal state and the subsequent recovery of superconductivity once $|q(t)|$ exceeds 1. At higher frequencies $\omega \gtrsim 0.1$, the superconducting state does not have enough time to disappear during the parts of the AC period in which $|q(t)|>1$, so that $\psi(t)$ at the minimum remains finite all the way to $q \rightarrow q_{d}$.

The calculated $Q_{d}(T)$ curves shown in Fig. 14 are similar to the TDGL results but generally fall below them: $Q_{d}(\Omega, T) \rightarrow \sqrt{2} Q_{c}=\sqrt{2\left(1-T / T_{c}\right)}$ at $\Omega \gtrsim \Omega_{c}(T)$ but $Q_{d}(\Omega, T) \rightarrow Q_{c}(T)$ at $\Omega \ll \Omega_{c}(T / 2)$. The temperature dependence of $\tau(T) \propto T^{-3}$ results in a crossover of $Q_{d}(T, \Omega)$ from $Q_{c}(T)$ to $\sqrt{2} Q_{c}(T)$ as $T$ decreases.

\section{Fixed $J(t)$}

Solutions of Eqs. (8)-(10) for $j=j_{a} \sin \omega t, \omega=0.1$, at $\tau=10$ and $\tau=100$ shown in Fig. 15 are qualitatively similar to that of $\psi(t)$ for a fixed $q(t)$. Here $\psi(t)$ vanishes abruptly at $j_{a}=j_{d}(\omega, T)$, the amplitude of oscillations of $\psi(t)$ essentially depends on $\omega$ and $\tau$, as shown in Fig. 16. The calculated $j_{d}=1.35 j_{c}$ at $\tau(T)=10$ turned out to be slightly smaller than the TDGL value, but at $\tau(T)=100$ both TDGL theory and Eqs. (8)-(10) give the same $j_{d}=\sqrt{2} j_{c}$. The dependencies of $j_{d}(\omega, \tau)$ on $\tau$ and $\omega$ shown in Fig. 17 appear similar to those for $q_{d}(\omega, \tau)$ in Fig. 12 and clearly demonstrate that $j_{d} \rightarrow \sqrt{2} j_{c}$ at $\omega \tau \gg 1$. The temperature dependence of $J_{d}(\Omega, T)$ shown in Fig. 18 is similar to the TDGL results only at $T \rightarrow T_{c}: J_{d}(\Omega, T) \rightarrow \sqrt{2} J_{c}(0)\left(1-T / T_{c}\right)^{3 / 2}$ at $\Omega \gtrsim$ $\Omega_{c}(T)$ and $J_{d}(\Omega, T) \rightarrow J_{c}(T)$ at $\Omega<\Omega_{c}(T)$. As $T$ decreases, the $J_{d}(\Omega, T)$ curves tend toward $J_{c}(T)$ even at $\Omega>\Omega_{c}(T)$. 


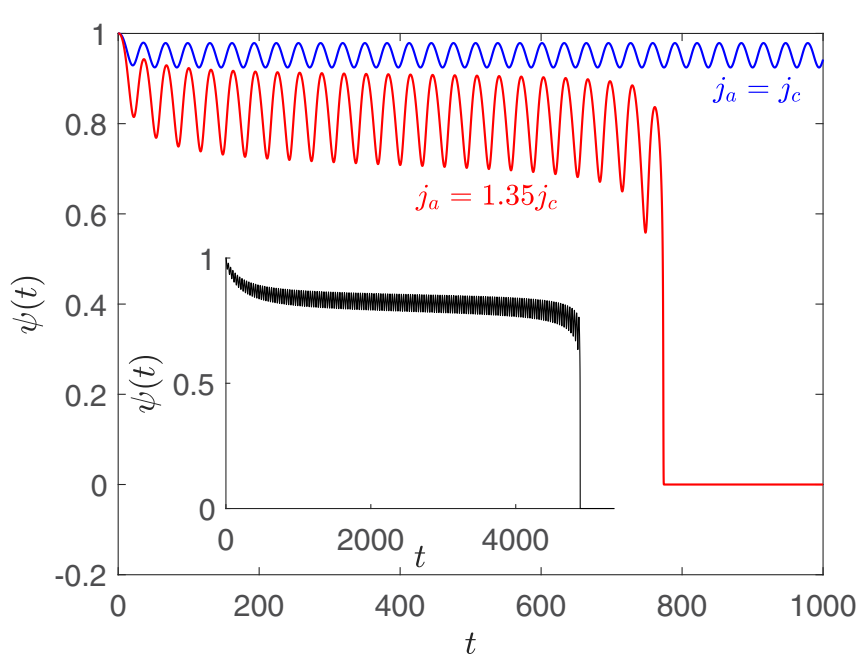

FIG. 15. Dynamics of $\psi(t)$ calculated at $j=j_{a} \sin \omega t, \omega=0.1$, $\tau=10, j_{a}=j_{c}$ and the critical current $j_{a}=1.35 j_{c}$ at which $\psi(t)$ vanishes abruptly. The inset shows $\psi(t)$ calculated at $\tau=100, \omega=$ 0.1 and $j_{a}=\sqrt{2} j_{c}$. All calculations were performed at $T=0.9 T_{c}$.

\section{NONLINEAR ELECTROMAGNETIC RESPONSE}

In this section we address an electromagnetic response of a nonequilibrium superconductor. For a nearly uniform current considered here, the linear response is quantified by a frequency-dependent complex conductivity,

$$
\mathbf{J}=\left(\sigma_{1}-i \sigma_{2}\right) \mathbf{E},
$$

where $\sigma_{1}(\Omega)$ describes a dissipative quasiparticle response, $\sigma_{2}(\Omega)=1 / \mu_{0} \Omega \lambda_{L}^{2}$ accounts for the Meissner effect, and $\lambda_{L}$ is the London penetration depth. Here $\sigma_{2}$ also determines the kinetic inductance $\mathcal{L}_{k}=\left(d \Omega \sigma_{2}\right)^{-1}=\mu_{0} \lambda_{L}^{2} / d$ per unit length of a film of thickness $d$ [46-50]. Using $\lambda_{L}^{2}(T)=$ $2 \hbar k_{B} T_{c} / \pi \mu_{0} \sigma_{0} \Delta_{0}^{2}$ near $T_{c}[21]$ yields

$$
\mathcal{L}_{k}=\frac{2 \hbar k_{B} T_{c}}{\pi \sigma_{0} d \Delta^{2}} .
$$
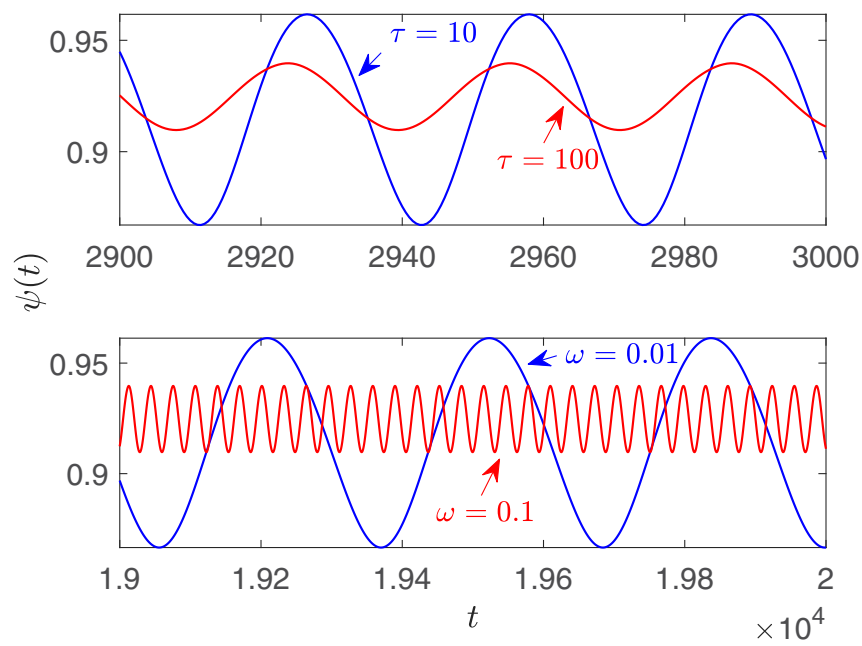

FIG. 16. Steady state oscillations of $\psi(t)$ calculated at $T=$ $0.9 T_{c}, j=j_{a} \sin \omega t, j_{a}=1.20 j_{c}$, and different $\tau$ at $\omega=0.1$ (top) and different $\omega$ at $\tau=100$ (bottom).
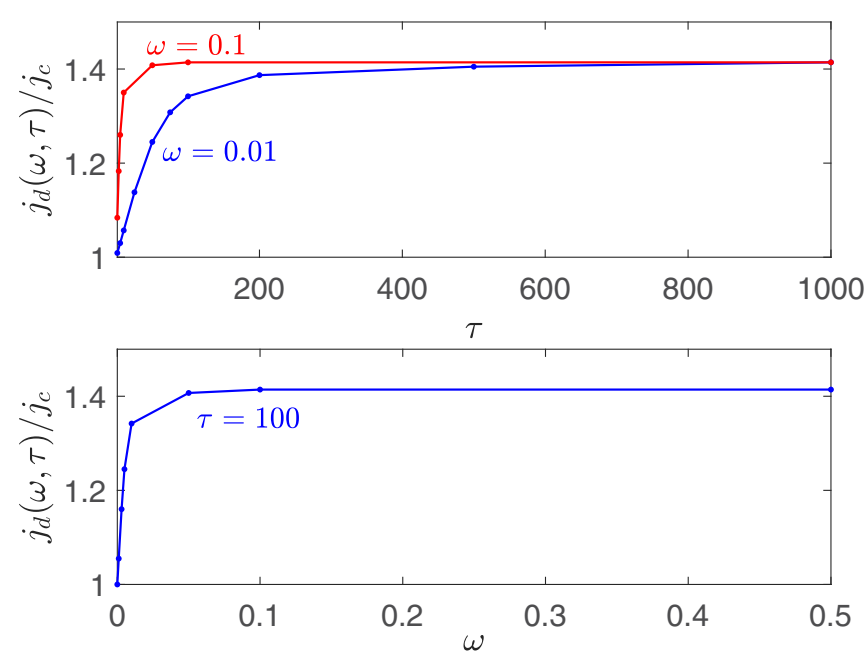

FIG. 17. Calculated dependencies of $j_{d}(\omega, \tau)$ on $\tau$ (top) and $\omega$ (bottom) at $T=0.9 T_{c}$. Here $j_{d}$ levels off at $\sqrt{2} j_{c}$ at $\omega \tau \gtrsim 1$.

At high current densities the conductivity $\sigma=\sigma_{1}-i \sigma_{2}$ depends on $Q(t)$, causing the nonlinear Meissner effect, intermodulation and generation of higher order harmonics of the electric field $E(t)$ in response to the AC current $J(t)=$ $J_{a} \sin \Omega t$ [40-45]. Defining the kinetic inductance by Eq. (14), where $\Delta(t)$ is given by the solutions of Eqs. (6) or Eqs. (8) and (9), we can expect strong oscillations of $\mathcal{L}_{k}(t)$ at large $J_{a}$ due to the nonequilibrium current pair breaking. Shown in Fig. 19 is the dynamics of $\mathcal{L}_{k}(t)$ calculated at a fixed $q(t)=q_{a} \sin \omega t$ with $q_{a}=0.9 \sqrt{2}, \omega=0.01$, and $\tau=100$. Here the amplitudes of $\mathcal{L}_{k}(t)$ increase with $q_{a}$ and diverge at $q_{a} \rightarrow q_{d}$, the peaks in $\mathcal{L}_{k}(t)$ getting higher as $\omega \tau$ decreases. Figure 19 also shows that the amplitudes of $\mathcal{L}_{k}(t)$ calculated from the full Eqs. (8)-(11) can be orders of magnitude higher as compared

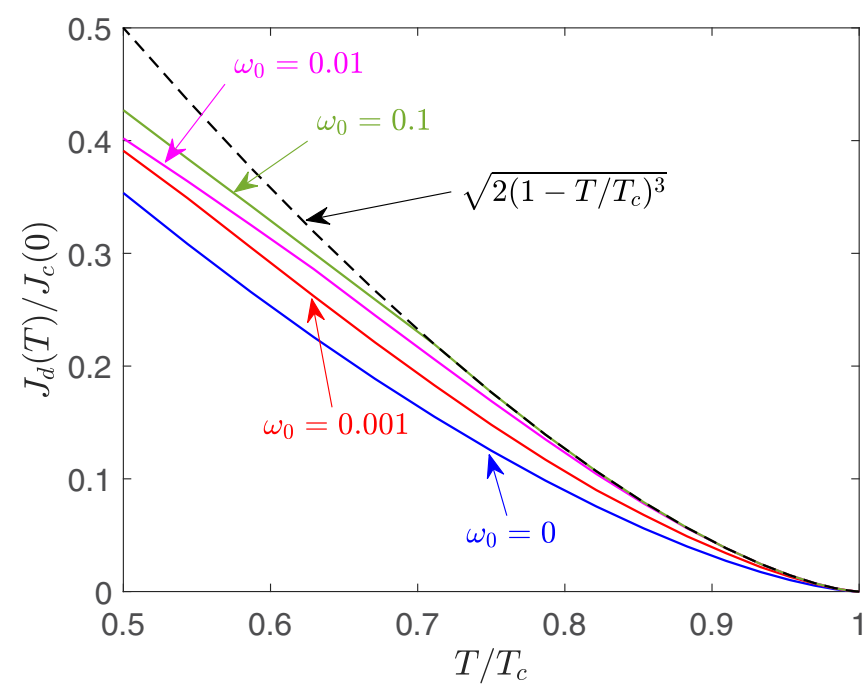

FIG. 18. $J_{d}(T)$ calculated from Eqs. (8)-(11) for $\tau_{0}=100$ at different $\omega_{0}=\pi \hbar \Omega / 8 k_{B} T_{c}$ where $\tau_{0}=\tau_{E}\left(T_{c}\right) \Delta_{0}(0)$, and $\Delta_{0}^{2}(0)=$ $8 \pi^{2} T_{c}^{2} / 7 \zeta(3)$. As $\Omega \gg \Omega_{c}(T)$, we have $J_{d}(T)=J_{c}(0) \sqrt{2}(1-$ $\left.T / T_{c}\right)^{3 / 2}$ at $T \rightarrow T_{c}$, however as $T$ decreases a crossover to $J_{c}(T)$ occurs even at $\Omega \geqslant \Omega_{c}(T)$. 


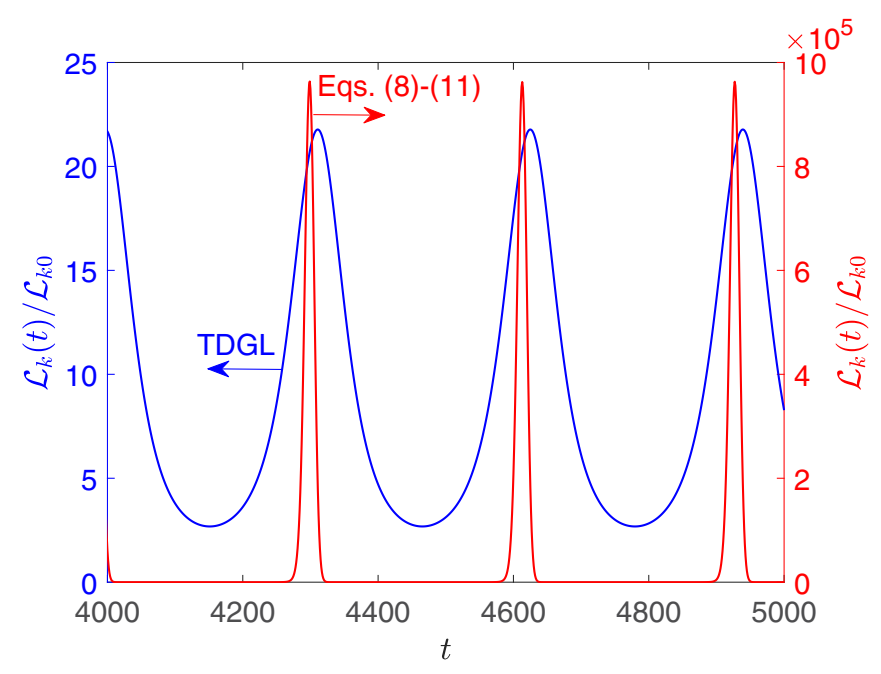

FIG. 19. Dynamics of $\mathcal{L}_{k}(t)$ in units of $\mathcal{L}_{k 0}=\pi \sigma_{0} d \Delta_{0}^{2} / 2 \hbar k_{B} T_{c}$ calculated from: (a) Eq. (6) and (b) Eqs. (8)-(11) at $T=0.9 T_{c}$ and $q(t)=q_{a} \sin \omega t$ with $q_{a}=0.9 \sqrt{2}, \omega=0.01$, and $\tau=100$. Notice large-amplitude oscillations of $\mathcal{L}_{k}(t)$ at small $\omega \tau$ and large $q_{a}$, the peaks in $\mathcal{L}_{k}(t)$ calculated from Eqs. (8)-(11) can be orders of magnitude larger than those obtained from Eq. (6).

to the TDGL results. This reflects larger amplitudes of oscillations of $\psi(t)$ calculated from Eqs. (8)-(11) and discussed above (see Fig. 11).

Shown in Fig. 20 is $\mathcal{L}_{k}(t)$ calculated from Eqs. (6) and (7) and Eqs. (8)-(11) at a fixed AC current $j=j_{a} \sin \omega t$ and $\tau=100$. Here $\mathcal{L}_{k}(t)$ can exhibit large-amplitude oscillations at small $\omega \tau$. The amplitudes of $\mathcal{L}_{k}(t)$ calculated from Eqs. (8)-(11) are larger than the TDGL results, although not by orders of magnitude.

The above calculations of $\mathcal{L}_{k}(t)$ pertain to low frequencies $\omega \tau \ll 1$ at which $\mathcal{L}_{k}(t)$ follows instantaneously to the time-varying order parameter $\Psi(t)$. Generally, the nonlinear electromagnetic response at a fixed $q(t)=q_{a} \sin \omega t$ causes

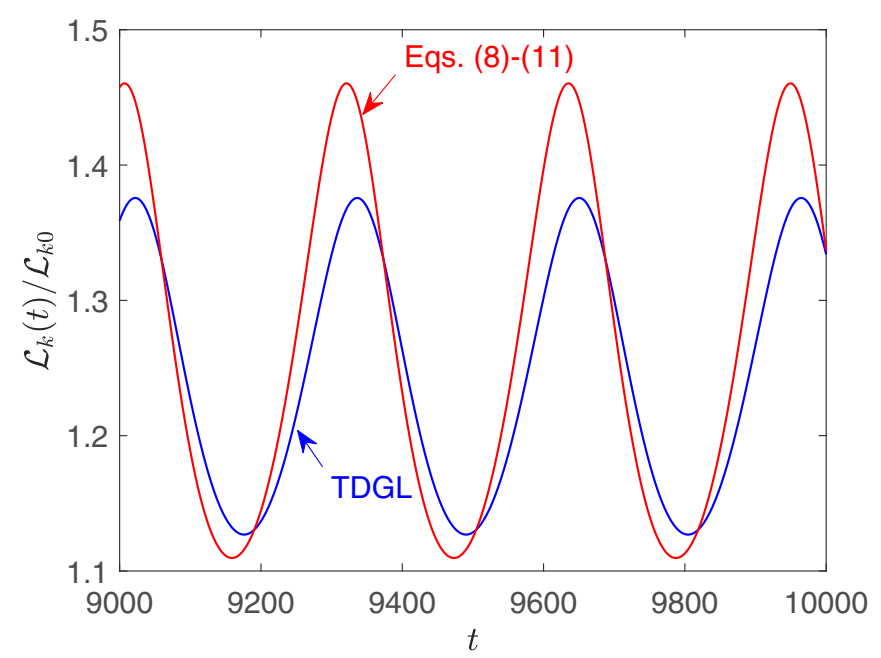

FIG. 20. Dynamics of $\mathcal{L}_{k}(t)$ calculated for a fixed current $j(t)=j_{a} \sin \omega t$ with $j_{a}=0.9 \sqrt{2} j_{c}, \omega=0.01$, and $\tau=100$ using: (a) Eqs. (6) and (7) and (b) Eqs. (8)-(11) at $T=0.9 T_{c}$.

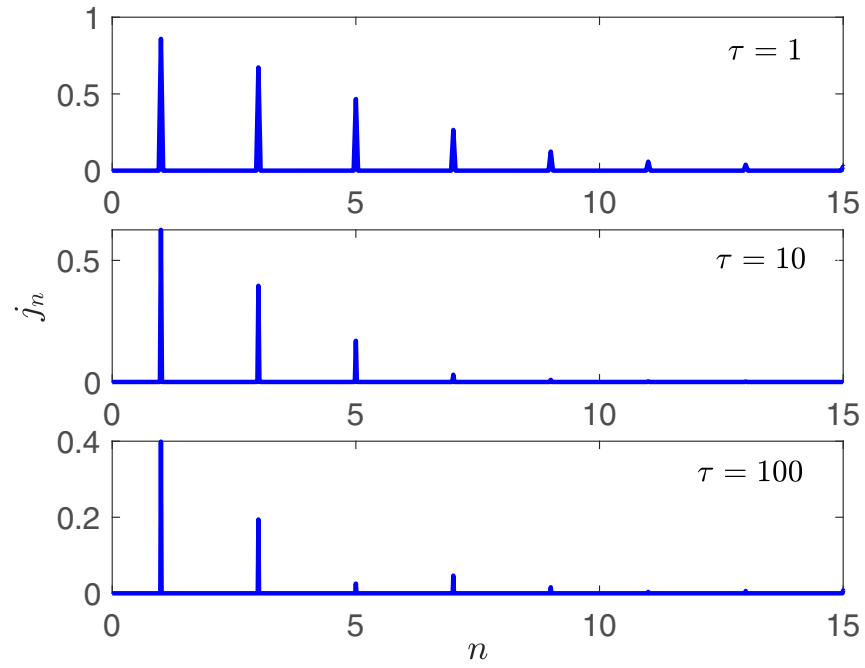

FIG. 21. Fourier spectra of the current amplitudes $j_{n}=$ $\sqrt{j_{1 n}^{2}+j_{2 n}^{2}}$ caused by $q(t)=q_{a} \sin \omega t$ calculated from Eqs. (8)-(10) for different $\tau$ at $T=0.9 T_{c}, q_{a}=0.95 \sqrt{2}$, and $\omega=0.1$. The Fourier amplitudes are peaked at $\omega_{n}=n \omega$ with $n=1,3,5, \ldots$.

generation of multiple current harmonics:

$$
j(t)=\sum_{n}\left[j_{1 n} \sin \omega_{n} t+j_{2 n} \cos \omega_{n} t\right] .
$$

Likewise, the AC current $j=j_{a} \sin \omega t$ produces multiple harmonics of the electric field $\varepsilon=\dot{q}$ :

$$
\varepsilon(t)=\sum_{n}\left[\varepsilon_{1 n} \sin \omega_{n} t+\varepsilon_{2 n} \cos \omega_{n} t\right] .
$$

Here the frequencies $\omega_{n}$ and the Fourier amplitudes $j_{1 n}\left(q_{a}\right)$, $j_{2 n}\left(q_{a}\right), \varepsilon_{1 n}\left(j_{a}\right)$, and $\varepsilon_{2 n}\left(j_{a}\right)$ are to be calculated selfconsistently from Eqs. (8)-(10), as shown below.

\section{A. Fixed $q(t)$}

Shown in Fig. 21 are the current Fourier spectra calculated at different $\tau$ at $q_{a}=0.95 \sqrt{2}$ and $\omega=0.1$. Here the multimode spectrum of $j(\omega)$ consisting of equidistant peaks at $\omega_{n}=n \omega, n=1,3,5, \ldots$ changes markedly as $\tau$ increases and the amplitudes of high-frequency harmonics diminish. The latter is consistent with the results of the previous sections which showed that at $\omega \tau \gg 1$ the amplitude of oscillations of superfluid density responsible for the generation of higher harmonics diminishes and the fundamental harmonic in $j(t)$ dominates. Here the nonequilibrium effects described by Eqs. (8) and (9) significantly increase the amplitudes of higher order harmonics as compared to the respective TDGL results.

Of particular interest is the dependence of the in-phase and out-of-phase parts of the amplitude of the main harmonic $j_{m}(t)=j_{1} \sin \omega t+j_{2} \cos \omega t$ on $q_{a}$, where $j_{2}$ determines the mean dissipative power $p=\omega q_{a} j_{2} / 2$. Shown in Fig. 22 are steady-state oscillations of $j(t)$ at $\tau=1$ and $\tau=100$. At $q_{a}=2^{-1 / 2}$ and $\tau=100$, the current response is nearly inphase with $q(t)$ but at $\tau=1$ the current has dips when $q(t)$ is maximum. The latter comes from pair-breaking effects which mostly reduce the superfluid density and the supercurrent when $q(t)$ reaches maximum. This effect becomes more 

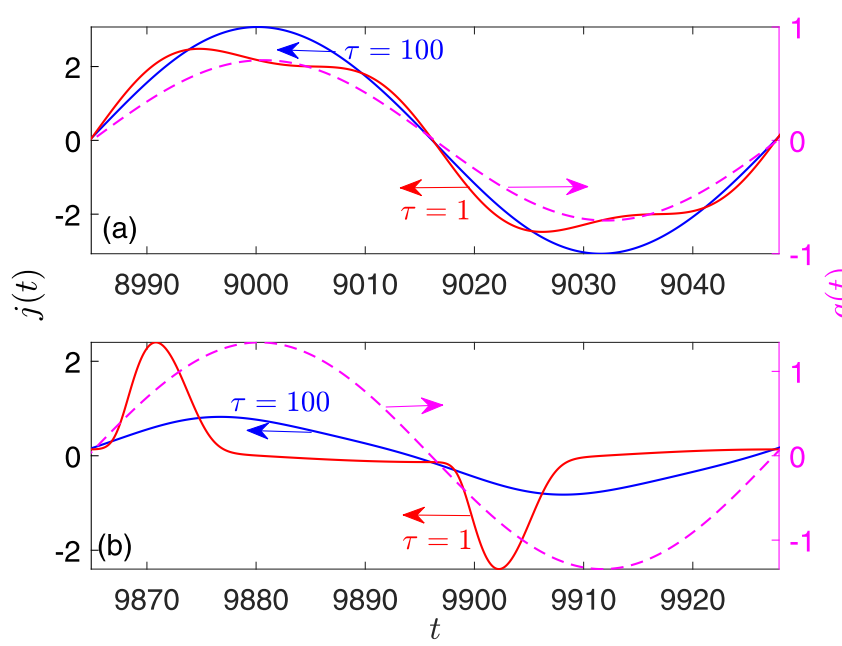

FIG. 22. Nonlinear current response $j(t)$ calculated at $q_{a}=$ $0.5 \sqrt{2}$ and $q_{a}=0.95 \sqrt{2}$ for two values of $\tau=1$ and $\tau=100$ at $T=0.9 T_{c}$. At $\tau=100$ the current is nearly in-phase with $q(t)$ at all $q_{a}$ 's. At $\tau=1$ the current response at large $q_{a}$ becomes almost evenly divided into the in-phase and out-of-phase parts.

pronounced for a larger amplitude $q_{a}=0.95 \sqrt{2}$ represented in Fig. 22(b). In this case $\psi(t)$ is much reduced during a considerable part of the AC period so $j_{1} \ll j_{2}$ and the current response becomes nearly ohmic.

The dependencies of the in-phase $j_{1}\left(q_{a}\right)$ and out-of-phase $j_{2}\left(q_{a}\right)$ amplitudes of the current main harmonic on $q_{a}$ are shown in Fig. 23 at $\tau=1$ and $\tau=100$. At $\tau=100$ the response current is mostly in-phase with $q(t)$ up to the critical $q_{a} \approx \sqrt{2}$, while at $\tau=1$, the out-of-phase part of $j_{m}(t)$ is essential and significantly increases with $q_{a}$ and the supercurrent decreases.

\section{B. Fixed $j(t)$}

To calculate the Fourier harmonics of the dimensionless electric field $\varepsilon(t)=E(t) / E_{0}=\partial q / \partial t$ with $E_{0}=\left(2 e \xi \tau_{\mathrm{GL}}\right)^{-1}$, we solved Eqs. (8)-(10) for $\psi(t)$ and $q(t)$ at a fixed AC current $j=j_{a} \sin \omega t$. Shown in Fig. 24 are the Fourier spectra $\varepsilon(\omega)$ at

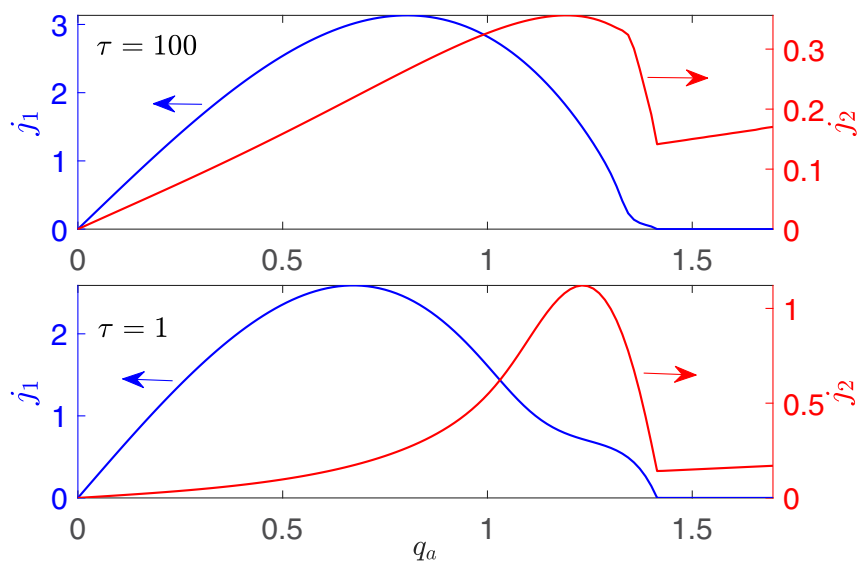

FIG. 23. The amplitudes $j_{1}\left(q_{a}\right)$ and $j_{2}\left(q_{a}\right)$ of the main current harmonic as functions of $q_{a}$ calculated from Eqs. (8)-(10) at $T=$ $0.9 T_{c}$ with $q(t)=q_{a} \sin \omega t$ at $\omega=0.1, \tau=1$, and $\tau=100$.
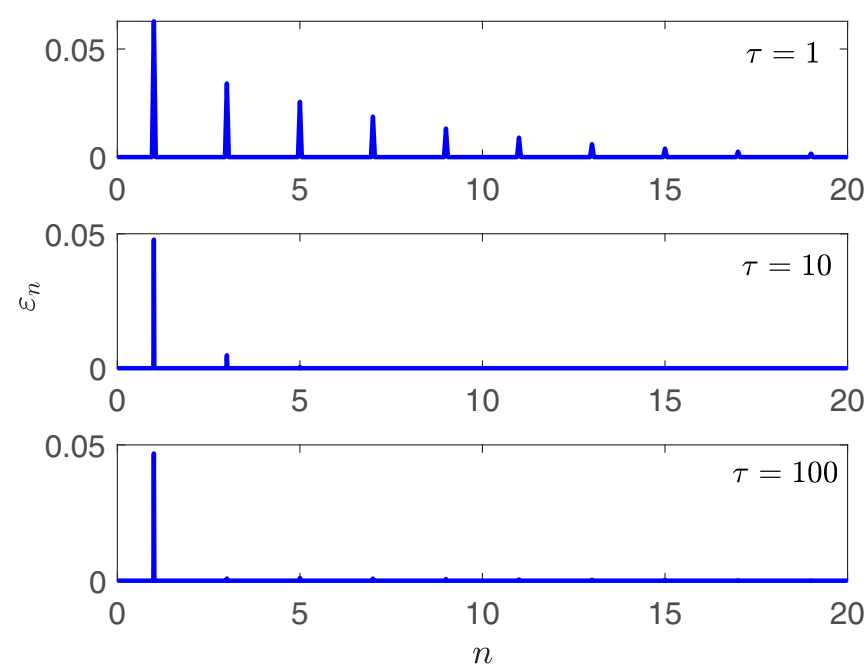

FIG. 24. Fourier spectra of the electric field $\varepsilon_{n}=\sqrt{\varepsilon_{1 n}^{2}+\varepsilon_{2 n}^{2}}$ in response to the AC current $j=j_{a} \sin \omega t$ calculated from Eqs. (8)(10) at $T=0.9 T_{c}, j_{a}=0.77 \sqrt{2} j_{c}, \omega=0.1$, and different $\tau$. The peaks in $\varepsilon_{n}$ occur at the odd multiples of $\omega$.

$j_{a}=0.77 \sqrt{2} j_{c}, \omega=0.1$, and different $\tau$. Like in the case of a fixed $q(t)$, the Fourier spectra of the electric field contain equidistant peaks at $\omega_{n}=n \omega$ with $n=1,3,5, \ldots$, the amplitudes of higher order harmonics decreasing as $\tau$ increases.

Figure 25 shows the in-phase and out-of-phase amplitudes $\varepsilon_{1}$ and $\varepsilon_{2}$ of the main harmonic $\varepsilon_{m}(t)=\varepsilon_{1} \sin \omega t+\varepsilon_{2} \cos \omega t$ as functions of $j_{a}$ at $\omega=0.1$ and two values of $\tau=1$ and $\tau=$ 100. Here $\varepsilon_{2}\left(j_{a}\right)$ describing the superfluid response dominates at all $j_{a}$ and is nearly linear in $j_{a}$, indicating that the dynamic differential resistivity $\rho_{2}=\partial \varepsilon_{2} / \partial j_{a}$ is weakly dependent on $j_{a}$ except for a sharp increase in a narrow region at $j_{a} \rightarrow j_{d}$ for both $\tau=1$ and $\tau=100$. By contrast, $\varepsilon_{1}\left(j_{a}\right)$ is linear in $j_{a}$ at $j_{a} \lesssim j_{d} / 2$ but then increases sharply as $j_{a}$ approaches $j_{d}$. The differential resistivities $\rho_{1}\left(j_{a}\right)=\partial \varepsilon_{1} / \partial j_{a}$ and $\rho_{2}\left(j_{a}\right)=$
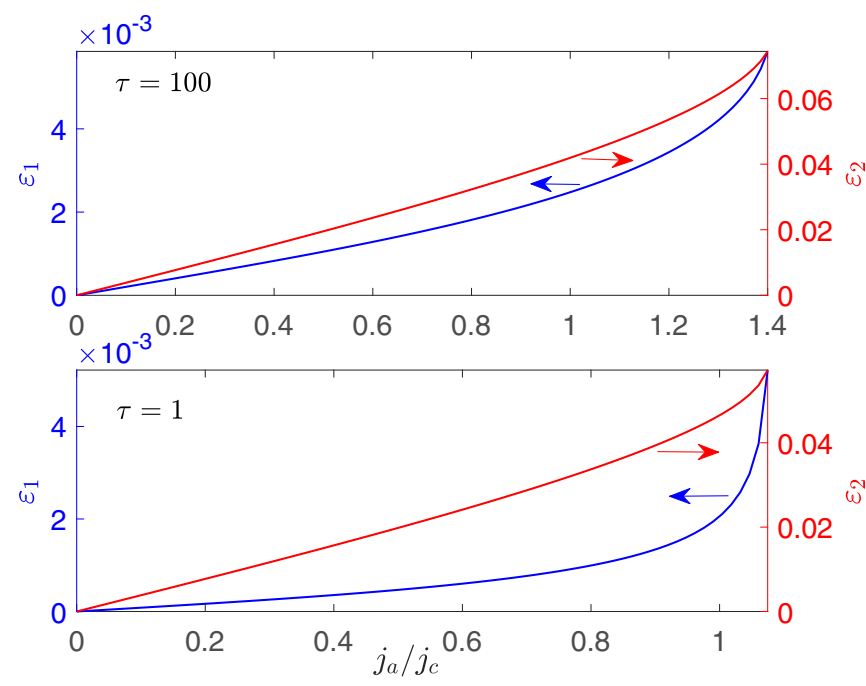

FIG. 25. The amplitudes $\varepsilon_{1}\left(j_{a}\right)$ and $\varepsilon_{2}\left(j_{a}\right)$ of the main electric field harmonic as functions of $j_{a}$ calculated from Eqs. (8)-(10) at $T=0.9 T_{c}$ with $j(t)=j_{a} \sin \omega t, \omega=0.1$, and $\tau=1$ and $\tau=100$. 

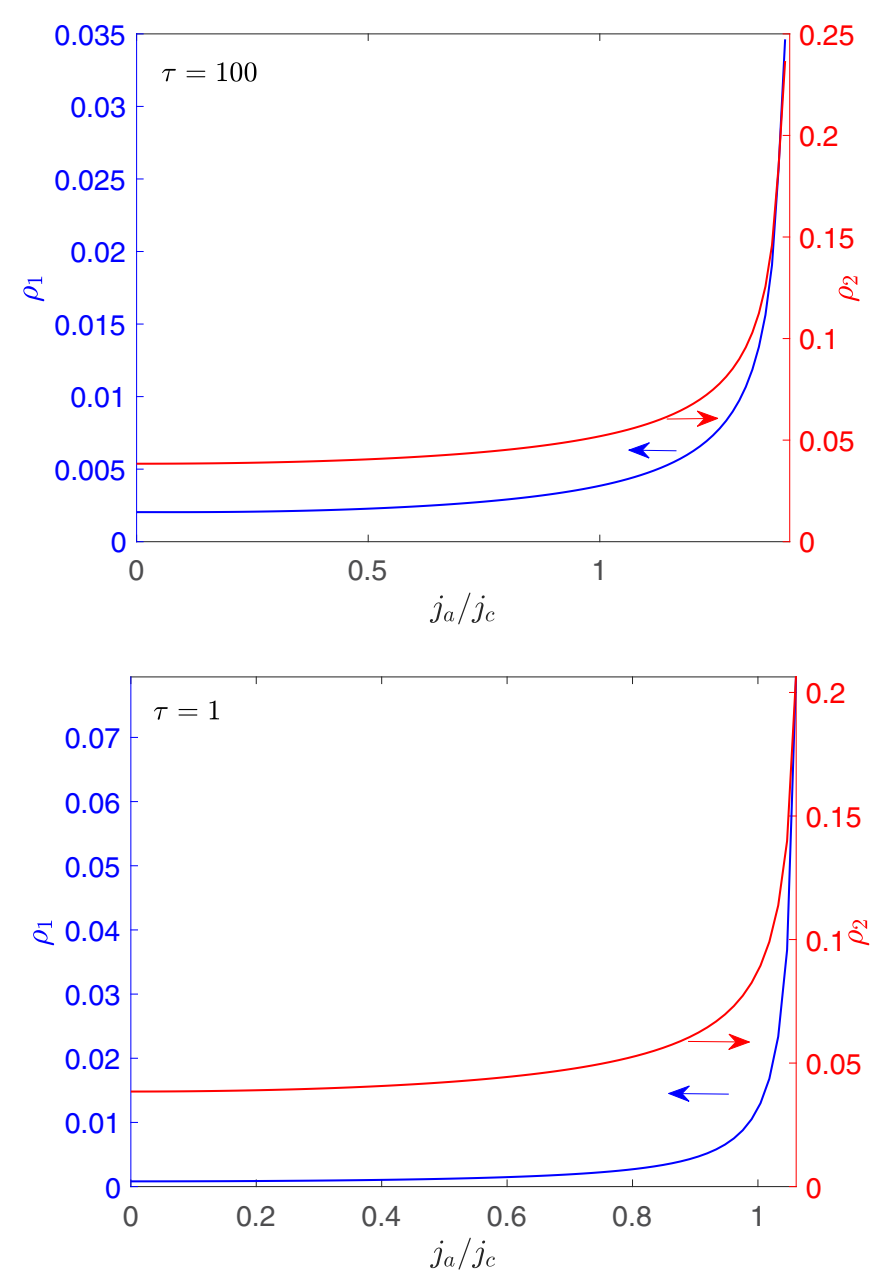

FIG. 26. Differential resistivities $\rho_{1}$ and $\rho_{2}$ as functions of $j_{a}$ calculated from Eqs. (8)-(10) at $T=0.9 T_{c}$ with $j(t)=j_{a} \sin \omega t$ at $\omega=0.1, \tau=1$, and $\tau=100$.

$\partial \varepsilon_{2} / \partial j_{a}$ as well as the resulting dissipated power $p=P / P_{0}=$ $\varepsilon_{1} j_{a} / 2$ as functions of $j_{a}$ where $P_{0}=E_{0} J_{0}$ are shown in Figs. 26 and 27, respectively. At $J>J_{d}$ the supercurrent density vanishes jumpwise, resulting in the ohmic response $J=\sigma_{0} E$ in the normal state. Notice that both $\rho_{1}$ and $\rho_{2}$ turned out to be much smaller than the normal state resistivity $\rho_{0}=1 / \sigma_{0}$ in the whole region of $0<J_{a}<J_{d}$.

\section{DISCUSSION}

In this work we address the breakdown of superconductivity by strong rf currents at $\hbar \Omega \ll \Delta_{0} \ll k_{B} T_{c}$. Here the deviation of the quasiparticle distribution function $f(E, t)$ from equilibrium is controlled by the amplitude of rf current and the inelastic electron-phonon scattering time $\tau_{E}$ which can be much larger than $\tau_{\mathrm{GL}}$ and the rf period $\Omega \tau_{E} \gg 1$. Because Eqs. (8)-(10) are applicable at $\hbar \Omega \ll k_{B} T_{c}$ [24-26], they do not describe a microwave stimulation of superconductivity which occurs at $\hbar \Omega \gtrsim k_{B} T$ [27]. Yet the kinetic equations (8)-(10) in which $\partial f / \partial E$ is replaced with its equilibrium value $\partial f_{0} / \partial E$ for a weak rf field $[25,26]$ can have spurious solutions corresponding to stimulated superconductivity. We

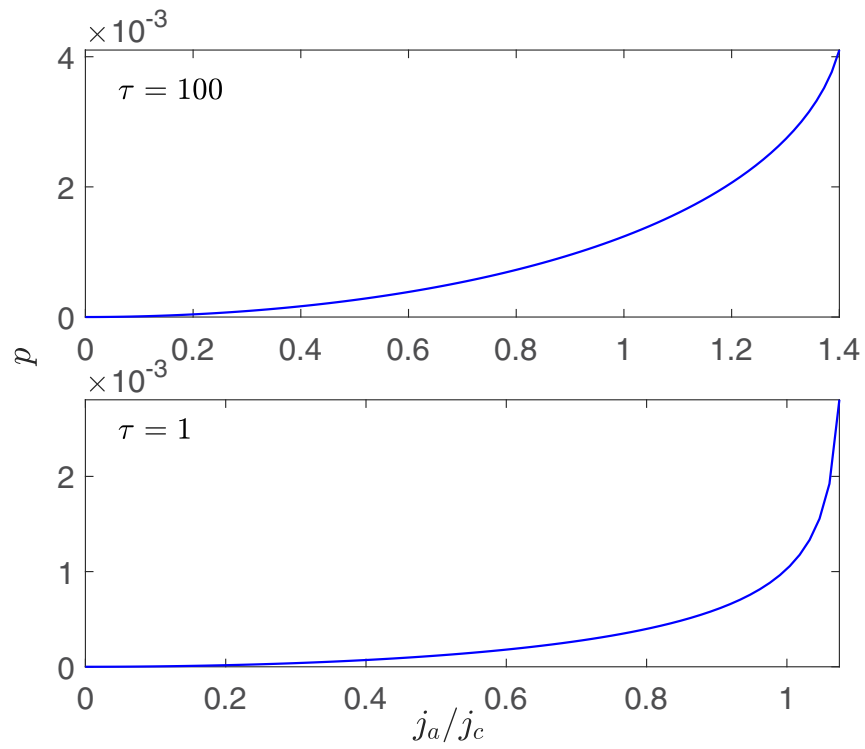

FIG. 27. AC power $p=\varepsilon_{1} j_{a} / 2$ as functions of $j_{a}$ calculated from Eqs. (8)-(10) at $T=0.9 T_{c}$ with $j(t)=j_{a} \sin \omega t$ at $\omega=0.1$ for $\tau=1$ and $\tau=100$.

did observe these solutions of the linearized equations (8)(10) but only at large rf amplitudes producing unphysical $\delta f(E, t)>1$. The results presented above are obtained using the Larkin-Ovchinnikov form of Eqs. (8)-(10) which include the exact $\partial f / \partial E$ [24]. In this case the nonequilibrium correction $\delta f(E, t)$ was always smaller than 1 and no stimulated superconductivity was observed.

The temperature and frequency dependencies of $Q_{d}$ and $J_{d}$ calculated from either the TDGL equations or Eqs. (8)(10) turned out to be similar. Namely, both $Q_{d}$ and $J_{d}$ tend to their respective static GL values at $\Omega \tau_{E} \ll 1$ and gradually increase with frequency, approaching the universal values $Q_{d} \rightarrow \sqrt{2} Q_{c}$ and $J_{d} \rightarrow \sqrt{2} J_{c}$ at $\max \left(\tau_{\mathrm{GL}}, \tau_{E}\right) \Omega \gg 1$. The physics of this effect is rather transparent: at $\Omega \tau_{E} \gg$ 1 , the pair potential $\psi(t)=\langle\psi\rangle+\delta \psi(t)$ undergoes smallamplitude rapid oscillations of $\delta \psi(t)$ around a mean value $\langle\psi\rangle$ which is determined by quasistatic equations with the time-averaged $\left\langle Q^{2}\right\rangle=Q_{a}^{2} / 2$. Thus, the solutions for the mean order parameter $\langle\psi\rangle$ disappear above the same pair-breaking critical value of $\left\langle Q^{2}\right\rangle$ as for a DC current. This result can also be used to evaluate the dynamic superheating field $H_{d}$ at which the Meissner state in a large- $\kappa$ superconductor becomes absolutely unstable:

$$
\begin{gathered}
H_{d}(T) \rightarrow H_{s}(T), \quad \Omega \tau_{E}(T) \ll 1, \\
H_{d}(T) \rightarrow \sqrt{2} H_{s}(T), \quad \Omega \tau_{E}(T) \gg 1, \\
H_{S}(T)=\left(\frac{\sqrt{5}}{3}+\frac{0.545}{\kappa}\right) H_{c}, \quad \kappa \gg 1,
\end{gathered}
$$

where $H_{S}(T)$ is the DC superheating field at $T \approx T_{c}$ [65]. At $\kappa \gg 1$ the screening current density varies slowly over $\xi$, so $Q(x, t)$ and $\Delta(x, t)$ are nearly independent of the coordinate $x$ perpendicular to the surface. 
The relation between the dynamic superheating field $H_{d}(T)$ and the DC superheating field $H_{s}(T)$ at low temperatures $T \ll T_{c}$ and frequencies $\hbar \Omega \ll k_{B} T_{c}$ has not yet been calculated from a microscopic theory. Yet based on the known dependence of the quasiparticle gap $\epsilon_{g}$ on the mean free path at $H=H_{s}$ [16], we can make qualitative conclusions [66] regarding the essential effect of impurities on $H_{d}(T)$ at $T \ll T_{c}$. In the dirty limit $l \ll \xi_{0}$ at $T \ll T_{c}$, the quasiparticle gap $\epsilon_{g}(H)$ diminishes as the field increases but remains finite all the way to $H_{s}$ at which $\epsilon_{g}\left(H_{s}\right) \approx 0.38 \Delta_{0}$ [16], where $H_{s}=0.84 H_{c}$ [14]. In this case the density of thermally activated quasiparticles remains exponentially small $n_{\mathrm{qp}}(T) \lesssim$ $n_{0}\left(\Delta_{0} / k_{B} T\right)^{1 / 2} \exp \left(-\epsilon_{g} / k_{B} T\right)$ in the entire field range of stability of the Meissner state, $0<H<H_{s}$. A low frequency field $\hbar \Omega \ll \Delta_{0}$ can produce nonequilibrium quasiparticles which can affect dissipative kinetic coefficients and the surface resistance [66], but the effect of an exponentially small density of quasiparticles at $T \ll T_{c}$ on the dynamics of the superconducting condensate would be negligible, unlike the case of $T \approx T_{c}$ considered in this work. As a result, the condensate at $T \ll T_{c}$ reacts nearly instantaneously to the rf field with $\Omega \ll \Delta_{0} / \hbar$, despite slow kinetics of sparse quasiparticles, so the superconductivity would be destroyed under the same pair-breaking condition as in the absence of quasiparticles. Thus, the dynamic superheating field $H_{d}$ of a dirty superconductor at $\hbar \Omega \ll \Delta_{0}$ and $T \ll T_{c}$ may be close to the static superheating field $H_{s} \approx 0.84 H_{c}$ even if $\Omega \tau_{E} \gg 1$.

For cleaner materials, the quasiparticle gap $\epsilon_{g}(H)$ vanishes before the DC depairing limit $H=H_{s}$ or $J=J_{c}$ is reached if $l \gtrsim 8.7 \xi_{0}$ [16]. In this case the density of quasiparticles at $H=H_{s}$ is no longer negligible so their slow kinetics at $T \ll T_{c}$ may increase $H_{d}$ relative to $H_{s}$ even at $\hbar \Omega \ll \Delta_{0}$. A similar situation can also occur in superconductors with a nanostructured surface [62] or inhomogeneous density of impurities [67], where the quasiparticle gap at the surface can be reduced by both the current pair breaking and the proximity effect. Complex effects of impurities on the electron-phonon and electron-electron energy relaxation have been a subject of many experimental investigations in recent years [68-71].

Our calculations of a nonlinear electromagnetic response of a nonequilibrium superconducting state show that the amplitudes of higher order harmonics diminish as the quasiparticle energy relaxation time $\tau_{E}$ increases. Typically $\tau_{E}$ near $T_{c}$ is about 2 orders of magnitude higher than $\tau_{\mathrm{GL}}$, except a narrow region of $T$ very close to $T_{c}$. Given that strong disorder can significantly reduce $\tau_{E}$ [68-71], one could expect that generation of higher order harmonics and intermodulation effects would be more pronounced in dirty superconductors. The moderate dependence of the dynamic differential resistivity $\rho_{2}\left(j_{a}\right)$ which defines a nonequilibrium kinetic inductance on $j_{a}$ shown in Fig. 26 is qualitatively similar to that of $\mathcal{L}_{k}\left(j_{a}\right)$ under the condition of the DC nonlinear Meissner effect $[40,41,43,45]$. At the same time, the dissipative differential resistivity $\rho_{1}\left(j_{a}\right)$ shown in Fig. 26 has a more pronounced dependence on $j_{a}$ than $\rho_{2}\left(j_{a}\right)$. Both $\rho_{1}\left(j_{a}\right)$ and $\rho_{2}\left(j_{a}\right)$ have strong peaks as $j_{a}$ approaches the dynamic depairing current density but remain much smaller than the normal state resistivity at low frequencies $\hbar \Omega \ll \Delta$. The nonlinearity of $\varepsilon\left(j_{a}\right)$ in a nonequilibrium state manifests itself in a strong dependence of the rf dissipated power on the current amplitude, as shown in Fig. 27.

\section{ACKNOWLEDGMENTS}

This work was supported by the U.S. Department of Energy under Grant DE-SC0010081-020 and by the National Science Foundation under Grant PHY 1734075.

\section{APPENDIX A: NONEQUILIBRIUM EQUATIONS}

The equations obtained in Refs. [23-26] for a nonequilibrium dirty $s$-wave superconductor at $T \approx T_{c}$ and $\Omega \ll \Delta_{0}$ include the quasistationary Usadel equation:

$\frac{D}{2}\left[\alpha(\nabla-2 i e \mathbf{A})^{2} \beta-\beta \nabla^{2} \alpha\right]=\left(\frac{1}{2 \tau_{E}}-i E\right) \beta-\Psi \alpha$,

where the normal and anomalous retarded Green's functions $\alpha(E)=N_{1}(E)+i R_{1}(E)$ and $\beta(E)=N_{2}+i R_{2}(E)$ satisfy $\alpha^{2}+\beta^{2}=1$. Equation (A1) is supplemented by the kinetic equations for the odd $f(E)$ and even $f_{1}(E)$ distribution functions of quasiparticles:

$$
\begin{gathered}
D \nabla \cdot\left[\left(N_{1}^{2}-R_{2}^{2}\right) \nabla \delta f\right]+2 D N_{2} R_{2} \mathbf{Q} \cdot\left(\nabla f_{1}-e \frac{\partial f}{\partial E} \frac{\partial \mathbf{A}}{\partial t}\right) \\
-N_{1}\left(\frac{\partial}{\partial t}+\frac{1}{\tau_{E}}\right) \delta f=R_{2} \frac{\partial f}{\partial E} \frac{\partial|\Psi|}{\partial t}, \\
D \nabla \cdot\left[\left(N_{1}^{2}+N_{2}^{2}\right)\left(\nabla f_{1}-e \frac{\partial f}{\partial E} \frac{\partial \mathbf{A}}{\partial t}\right)\right] \\
+2 D N_{2} R_{2} \mathbf{Q} \cdot \nabla \delta f-N_{1}\left(\frac{\partial}{\partial t}+\frac{1}{\tau_{E}}\right)\left(f_{1}+e \varphi \frac{\partial f}{\partial E}\right) \\
-N_{2}|\Psi|\left(2 f_{1}+\frac{\partial f}{\partial E} \frac{\partial \theta}{\partial t}\right)=0,
\end{gathered}
$$

where $f=f_{0}+\delta f$ and $f_{0}=\tanh (E / 2 T)$.

The equations for $\Psi(\mathbf{r}, t)=\Delta \exp (-i \theta)$ and $\mathbf{J}(\mathbf{r}, t)$ are expressed in terms of $N_{1,2}, R_{1,2}, \delta f$ and $f_{1}$ as follows [25,26]:

$$
\begin{gathered}
{\left[\frac{\pi}{8 T_{c} \epsilon} \frac{\partial}{\partial t}-\frac{1}{\Delta \epsilon} \int_{0}^{\infty} d E\left(R_{2} \delta f+i N_{2} f_{1}\right)\right] \Psi} \\
=\xi^{2}(\nabla-2 i e \mathbf{A})^{2} \Psi+\left(1-\frac{\Delta^{2}}{\Delta_{0}^{2}}\right) \Psi, \\
\mathbf{J}=\frac{\pi \sigma_{0}}{4 e T_{c}} \Delta^{2} \mathbf{Q}+\frac{\sigma_{0}}{e} \int_{0}^{\infty} d E\left[\left(N_{1}^{2}+N_{2}^{2}\right)\left(\nabla f_{1}-e \frac{\partial f}{\partial E} \frac{\partial \mathbf{A}}{\partial t}\right)\right. \\
\left.+2 N_{2} R_{2} \mathbf{Q} \delta f\right] .
\end{gathered}
$$

If $\delta f(E, r, t)$ and $\Psi(r, t)$ vary slowly over $\tau_{E}, \xi$, and $L_{E}=$ $\left(D \tau_{E}\right)^{1 / 2}$, the derivatives in Eqs. (A2) and (A3) can be neglected. In this local equilibrium approximation Eqs. (A1)(A5) reduce to Eqs. (3) and (4) $[25,26]$.

If the spatial derivatives in Eqs. (A1)-(A5) are negligible we readily obtain $f_{1}=-e \varphi \partial f / \partial E$ and $\Phi=-2 e \varphi+$ $\partial \theta / \partial t=0$ from Eq. (A3), giving $\nabla f_{1}-e(\partial f / \partial E)(\partial \mathbf{A} / \partial t)=$ $1 / 2(\partial f / \partial E)(\partial \mathbf{Q} / \partial t)$. In turn, Eq. (A1) reduces to the quartic 
equation:

$$
\begin{aligned}
& \alpha^{4}-\mathcal{R} \alpha^{3}+\mathcal{S} \alpha^{2}+\mathcal{R} \alpha-\frac{\mathcal{R}^{2}}{4}=0, \\
& \mathcal{R}=\frac{2(u / \epsilon)^{1 / 2}(i E-1 / 2 \tau)}{q^{2}}, \\
& \mathcal{S}=\frac{\mathcal{R}^{2}}{4}\left[\frac{\psi^{2}}{(i E-1 / 2 \tau)^{2}}+1\right]-1 .
\end{aligned}
$$

The relevant solution of Eq. (A6) is given by

$$
\alpha(E)=\frac{\mathcal{R}}{4}+\mathcal{E}+\frac{1}{2} \sqrt{-4 \mathcal{E}^{2}-2 \mathcal{A}-\frac{\mathcal{B}}{\mathcal{E}}},
$$

where

$$
\begin{aligned}
\mathcal{A} & =\mathcal{S}-\frac{3 \mathcal{R}^{2}}{8}, \quad \mathcal{B}=8 \mathcal{R}+4 \mathcal{R S}-\frac{\mathcal{R}^{3}}{8} \\
\mathcal{C} & =2 \mathcal{S}^{3}+27 \mathcal{R}^{2} \mathcal{S}+27 \mathcal{R}^{2}-\frac{27 \mathcal{R}^{4}}{4} \\
\mathcal{D} & =\left[\frac{1}{2}\left(\mathcal{C}+\sqrt{\mathcal{C}^{2}-4 \mathcal{S}^{6}}\right)\right]^{1 / 3} \\
\mathcal{E} & =\frac{1}{2} \sqrt{-\frac{2 \mathcal{A}}{3}+\frac{1}{3}\left(\mathcal{D}+\frac{\mathcal{S}^{2}}{\mathcal{D}}\right)}
\end{aligned}
$$

\section{APPENDIX B: HIGH-FREQUENCY LIMIT, $\omega \tau$ 》 1}

At high frequencies $\psi(t)=\psi+\delta \psi(t)$ has a smallamplitude oscillating component $\delta \psi(t) \ll \psi$ around a mean value $\psi$ so that $\langle\delta \psi\rangle=0$, where $\langle\cdots\rangle$ denotes time averaging. In this case Eqs. (6) and (7) can be solved by the standard methods which have been developed for dynamic equations with rapidly oscillating parameters [72,73].

\section{Fixed $Q(t)$}

For a fixed $q(t)=q_{a} \sin \omega t$ we expand Eq. (6) up to quadratic terms in $\delta \psi$ and average over the rf period:

$$
\begin{gathered}
r \dot{\psi}=\left(1-\left\langle q^{2}\right\rangle\right) \psi-\psi^{3}+\langle h \delta \psi\rangle-3\left\langle\delta \psi^{2}\right\rangle \psi, \\
h(t)=\left\langle q^{2}\right\rangle-q^{2}(t)=\frac{q_{a}^{2}}{2} \cos 2 \omega t,
\end{gathered}
$$

where $r=\left(1+4 \tau^{2} \psi^{2}\right)^{1 / 2},\left\langle q^{2}\right\rangle=q_{a}^{2} / 2$, and $\langle\delta \dot{\psi} \delta \psi\rangle=0$.

The dynamic equation for $\delta \psi(t)$ is obtained by expanding Eq. (6) up to linear terms in $\delta \psi$ :

$$
r \psi \dot{\psi}-g \delta \psi=h(t) \psi, \quad g=1-q_{a}^{2} / 2-3 \psi^{2} .
$$

The solution of Eq. (B3) is then

$$
\begin{gathered}
\delta \psi(t)=A \cos 2 \omega t+B \sin 2 \omega t, \\
A=-\frac{q_{a}^{2} g \psi}{2\left(4 \omega^{2} r^{2}+g^{2}\right)}, \quad B=\frac{q_{a}^{2} \omega r \psi}{4 \omega^{2} r^{2}+g^{2}} .
\end{gathered}
$$

From Eqs. (B1) and (B4) we obtain the following selfconsistency equation for $\psi(t)$ :

$$
r \dot{\psi}=\left(1-\frac{q_{a}^{2}}{2}\right) \psi-\psi^{3}+\frac{q_{a}^{2} A}{4}-\frac{3}{2} \psi\left(A^{2}+B^{2}\right) .
$$

At $4 \omega^{2} r^{2} \gg g^{2}$, Eqs. (B5) and (B6) reduce to

$$
r \dot{\psi}=\left(1-\frac{q_{a}^{2}}{2}\right)\left(1-\frac{q_{a}^{4}}{32 \omega^{2} r^{2}}\right) \psi-\psi^{3}
$$

Hence, the mean steady-state $\psi$ is given by

$$
\psi=\left(1-\frac{q_{a}^{2}}{2}\right)^{1 / 2}\left(1-\frac{q_{a}^{4}}{64 \omega^{2} r^{2}}\right) .
$$

This state is stable with respect to small perturbations of $\psi(t)$ if $q_{a}<q_{d}=\sqrt{2}$.

\section{Fixed $J(t)$}

For a fixed $j(t)=j_{a} \sin \omega t$, we linearize Eq. (7) with respect to an oscillating correction $\delta \psi(t) \ll 1$ :

$$
j_{a} \sin \omega t=q u \psi^{2}+2 u \psi q \delta \psi+\dot{q} .
$$

Setting here $q(t)=q_{1} \sin \omega t+q_{2} \cos \omega t \quad$ and $\quad \delta \psi=$ $A \cos 2 \omega t+B \sin 2 \omega t$, we obtain $\langle q \delta \psi\rangle=0$, and $q(t)=-\left(j_{a} / u \psi^{2}\right) \sin \omega t$ in leading order in $\omega / u \ll 1$ and $(\omega r)^{-2} \ll 1$. Substituting this $q(t)$ into Eq. (6) and averaging gives the equation for the mean $\psi(t)$ :

$$
\left(1+4 \tau^{2} \psi^{2}\right)^{1 / 2} \dot{\psi}=\left(1-\frac{j_{a}^{2}}{2 u^{2} \psi^{4}}\right) \psi-\psi^{3}
$$

The right-hand side of Eq. (B10) has the GL form for a fixed current except that the time averaging of $\left\langle q^{2}(t)\right\rangle=j_{a}^{2} / 2 u^{2} \psi^{4}$ reduces the current pair-breaking term in half as compared to the DC current. As a result,

$$
j_{a}^{2}=2 u^{2} \psi^{4}\left(1-\psi^{2}\right)
$$

Stability of the above steady state with respect to slow perturbations $\psi_{1}(t)$ can be addressed by setting $\psi(t)=\psi+\psi_{1}(t)$ and linearizing Eq. (B10) with respect to $\psi_{1}$ :

$$
r \dot{\psi_{1}}=\left[1+\frac{3 j_{a}^{2}}{2 u^{2} \psi^{4}}-3 \psi^{2}\right] \psi_{1}
$$

Hence, $\psi_{1} \propto \exp (\gamma t)$, where the decrement $\gamma$ is given by

$$
\gamma=\frac{2}{r}\left(2-3 \psi^{2}\right)
$$

Here $j_{a}^{2}$ in Eq. (B12) was expressed in terms of $\psi^{2}$ using Eq. (B11). This state becomes unstable $(\gamma>0)$ at $j_{d}=\sqrt{2} j_{c}$ for which $j_{a}(\psi)$ reaches maximum at $\psi^{2}=2 / 3$. 
[1] M. Tinkham, Introduction to Superconductivity, 2nd ed. (McGraw-Hill, New York, 1995).

[2] V. L. Ginzburg, Dokl. Akad. Nauk SSSR 118, 464 (1958) [Sov. Phys.-Doklady 3, 102 (1958)].

[3] R. H. Parmenter, RCA Rev. 26, 23 (1962).

[4] J. Bardeen, Rev. Mod. Phys. 34, 667 (1962).

[5] K. Maki, Prog. Theor. Phys. 29, 333 (1963).

[6] K. Maki, Gapless superconductivity, in Superconductivity, edited by R. D. Parks (Marcel Dekker, New York, 1969).

[7] M. Yu. Kupriyanov and V. F. Lukichev, Fiz. Nizk. Temp. 6, 445 (1980) [Sov. J. Low Temp. Phys. 6, 210 (1980)].

[8] E. J. Nicol and J. P. Carbotte, Phys. Rev. B 43, 10210 (1991).

[9] M. N. Kunchur, D. K. Christen, C. E. Klabunde, and J. M. Phillips, Phys. Rev. Lett. 72, 752 (1994).

[10] N. M. Kunchur, J. Phys. Condens. Matter 16, R1183 (2004).

[11] V. Rouco, C. Navau, N. Del-Valle, D. Massarotti, G. P. Papari, D. Stornaiuolo, X. Obradors, T. Puig, F. Tafuri, A. Sanchez, and A. Palau, Nano Lett. 19, 4174 (2019).

[12] J. Matricon and D. Saint-James, Phys. Lett. A 24, 241 (1967).

[13] S. J. Chapman, SIAM J. Appl. Math. 55, 1233 (1995).

[14] V. P. Galaiko, Zh. Eksp Teor. Fiz. 50, 717 (1966) [Sov. Phys. JETP 23, 475 (1966)].

[15] G. Catelani and J. P. Sethna, Phys. Rev. B 78, 224509 (2008).

[16] F. P.-J. Lin and A. Gurevich, Phys. Rev. B 85, 054513 (2012).

[17] W. Belzig, C. Bruder, and G. Schön, Phys. Rev. B 53, 5727 (1996).

[18] A. L. Fauchere and G. Blatter, Phys. Rev. B 56, 14102 (1997).

[19] W. Belzig, C. Bruder, and A. L. Fauchere, Phys. Rev. B 58, 14531 (1998).

[20] A. V. Galaktionov and A. D. Zaikin, Phys. Rev. B 67, 184518 (2003).

[21] N. B. Kopnin, Theory of Nonequilibrium Superconductivity (Oxford University Press, Oxford, England, 2001).

[22] S. B. Kaplan, C. C. Chi, D. N. Langenberg, J. J. Chang, S. Jafarey, and D. J. Scalapino, Phys. Rev. B 14, 4854 (1976).

[23] A. Schmid and G. Schön, J. Low Temp. Phys. 20, 207 (1975).

[24] A. I. Larkin and Yu. N. Ovchinnikov, Zh. Eksp. Teor. Fiz. 73, 299 (1977) [Sov. Phys. JETP 46, 1 (1977)].

[25] L. Kramer and R. J. Watts-Tobin, Phys. Rev. Lett. 40, 1041 (1978).

[26] R. J. Watts-Tobin, Y. Krähenbühl, and L. Kramer, J. Low Temp. Phys. 42, 459 (1981).

[27] G. M. Eliashberg and B. I. Ivlev, Nonequilibrium Superconductivity, edited by D. N. Langenberg and A. I. Larkin (Elsevier, Amsterdam, 1986), p. 211.

[28] P. Fulde, Phys. Rev. 137, A783 (1965).

[29] A. Anthore, H. Pothier, and D. Esteve, Phys. Rev. Lett. 90, 127001 (2003).

[30] P. K. Day, H. G. Leduc, B. A. Mazin, A. Vayonakis, and J. Zmuidzinas, Nature (London) 425, 817 (2003).

[31] J. Zmuidzinas, Rev. Condens. Matter Phys. 3, 169 (2012).

[32] H. Padamsee, J. Knobloch, and T. Hays, RF Superconductivity for Accelerators (John Wiley, New York, 1998).

[33] A. Gurevich, Supercond. Sci. Technol. 30, 034004 (2017).

[34] T. Yogi, G. J. Dick, and J. E. Mercereau, Phys. Rev. Lett. 39, 826 (1977).

[35] S. Posen, N. Valles, and M. Liepe, Phys. Rev. Lett. 115, 047001 (2015).

[36] W. J. Skocpol, M. R. Beasley, and M. Tinkham, J. Low Temp. Phys. 16, 145 (1974).
[37] B. I. Ivlev and N. B. Kopnin, Adv. Phys. 33, 47 (1984).

[38] R. Tidecks, Current-Induced Nonequilibrium Phenomena in Quasi-One-Dimensional Superconductors (Springer, Berlin, 2006), Vol. 121.

[39] D. Y. Vodolazov and F. M. Peeters, Phys. Rev. B 81, 184521 (2010).

[40] S. K. Yip and J. A. Sauls, Phys. Rev. Lett. 69, 2264 (1992); D. Xu, S. K. Yip, and J. A. Sauls, Phys. Rev. B 51, 16233 (1995).

[41] T. Dahm and D. J. Scalapino, J. Appl. Phys. 81, 2002 (1997); Phys. Rev. B 60, 13125 (1999).

[42] W. Hu, A. S. Thanawalla, B. J. Feenstra, F. C. Wellstood, and S. M. Anlage, Appl. Phys. Lett. 75, 2824 (1999).

[43] M. R. Li, P. J. Hirschfeld, and P. Wölfle, Phys. Rev. Lett. 81, 5640 (1998); Phys. Rev. B 61, 648 (2000).

[44] D. E. Oates, J. Supercond. Novel Magn. 20, 3 (2007).

[45] N. Groll, A. Gurevich, and I. Chiorescu, Phys. Rev. B 81, 020504(R) (2010).

[46] R. Meservey and P. M. Tedrow, J. Appl. Phys. 40, 2028 (1969).

[47] J. R. Clem and E. H. Brandt, Phys. Rev. B 72, 174511 (2005).

[48] G. Via, C. Navau, and A. Sanchez, J. Appl. Phys. 113, 093905 (2013)

[49] A. J. Annunziata, D. F. Santavicca, L. Frunzio, G. Catelani, M. J. Rooks, A. Frydman, and D. E. Prober, Nanotechnology 21, 445202 (2010).

[50] K. Enpuku, H. Moritaka, H. Inokuchi, T. Kisu, and M. Takeo, Jpn. J. Appl. Phys. 34, L675 (1995).

[51] D. E. McCumber and B. I. Halperin, Phys. Rev. B 1, 1054 (1970).

[52] K. Yu. Arutyunov, D. S. Golubev, and A. D. Zaikin, Phys. Rep. 464, 1 (2008).

[53] J. E. Mooij and Yu. V. Nazarov, Nat. Phys. 2, 169 (2006).

[54] M. Sahu, M-H. Bae, A. Rogachev, D. Pekker, T-C. Wei, N. Shah, P. M. Goldbart, and A. Bezryadin, Nat. Phys. 5, 503 (2009).

[55] R. Rangel and L. Kramer, J. Low Temp. Phys. 74, 163 (1989).

[56] D. Y. Vodolazov, A. Elmuradov, and F. M. Peeters, Phys. Rev B 72, 134509 (2005).

[57] L. Kramer and R. Rangel, J. Low Temp. Phys. 57, 391 (1984).

[58] N. W. Ashkroft and N. D. Mermin, Solid State Physics (Holt, Rinehart and Winston, Philadelphia, 1976).

[59] J. P. Carbotte, Rev. Mod. Phys. 62, 1027 (1990).

[60] R. C. Dynes, V. Narayanamurti, and J. P. Garno, Phys. Rev. Lett. 39, 229 (1977).

[61] J. Zasadzinski, Tunneling spectroscopy of conventional and unconventional superconductors. in The Physics of Superconductors, edited by K. H. Bennemann and J. B. Ketterson (Springer, New York, 2003), Chap. 15, p. 591.

[62] T. Kubo and A. Gurevich, Phys. Rev. B 100, 064522 (2019).

[63] W. E. Schiesser, The Numerical Method of Lines: Integration of Partial Differential Equations (Academic, San Diego, 1991).

[64] L. F. Shampine and M. K. Gordon, Computer Solution of Ordinary Differential Equations: The Initial Value Problem (W. H. Freeman, San Francisco, 1975).

[65] M. K. Transtrum, G. Catelani, and J. P. Sethna, Phys. Rev. B 83, 094505 (2011).

[66] A. Gurevich, Phys. Rev. Lett. 113, 087001 (2014).

[67] V. Ngampruetikorn and J. A. Sauls, Phys. Rev. Res. 1, 012015(R) (2019).

[68] A. Leo, G. Grimaldi, R. Citro, A. Nigro, S. Pace, and R. P. Huebener, Phys. Rev. B 84, 014536 (2011). 
[69] M. V. Sidorova, A. G. Kozorezov, A. V. Semenov, Yu. P. Korneeva, M. Yu. Mikhailov, A. Yu. Devizenko, A. A. Korneev, G. M. Chulkova, and G. N. Goltsman, Phys. Rev. B 97, 184512 (2018).

[70] L. Zhang, L. You, X. Yang, J. Wu, C. Lv, Q. Guo, W. Zhang, H. Li, W. Peng, Z. Wang, and X. Xie, Sci. Rep. 8, 1486 (2018).
[71] Yu. P. Korneeva, N. N. Manova, I. N. Florya, M. Yu. Mikhailov, O. V. Dobrovolskiy, A. A. Korneev, and D. Yu. Vodolazovs, Phys. Rev. Appl. 13, 024011 (2020).

[72] L. D. Landau and E. M. Lifshitz, Mechanics (Elsevier, Boston, 1976).

[73] N. N. Bogoliubov and Y. A. Mitropolski, Asymptotic Methods in the Theory of Nonlinear Oscillations (Gordon and Breach, New York, 1961). 